Vietnam Journal of Mechanics, VAST, Vol.40, No. 4 (2018), pp. 349-375

DOI: https://doi.org/10.15625/0866-7136/13499

\title{
A GENERALISATION TO COHESIVE CRACKS EVOLUTION UNDER EFFECTS OF NON-UNIFORM STRESS FIELD
}

\author{
Tuan-Hiep Pham ${ }^{1, *}$, Jérôme Laverne ${ }^{2}$, Jean-Jacques Marigo ${ }^{3}$ \\ ${ }^{1}$ CEA, Université Paris-Saclay, DEN, Service d'Études des Matériaux Irradiés, France \\ ${ }^{2}$ EDF RED, Laboratoire National d'Hydraulique et Environnement, France \\ ${ }^{3}$ CNRS, Ecole Polytechnique, Laboratoire de Mécanique des Solides, France \\ *E-mail: tuan-hiep.pham@cea.fr \\ Received Frebuary 07, 2018
}

\begin{abstract}
The aim of the present work is to study the stabilizing effect of the nonuniformity of the stress field on the cohesive cracks evolution in two-dimensional elastic structures. The crack evolution is governed by Dugdale's or Barenblatt's cohesive force models. We distinguish two stages in the crack evolution: the first one where all the crack is submitted to cohesive forces, followed by a second one where a non cohesive part appears. Assuming that the material characteristic length $d_{c}$ associated with the cohesive model is small by comparison to the dimension $L$ of the body, we develop a two-scale approach, and using the complex analysis method, we obtain the entire crack evolution with the loading in a closed form for the Dugdale's case and in semi-analytical form for the Barenblatt's case. In particular, we show that the propagation is stable during the first stage, but becomes unstable with a brutal jump of the crack length as soon as the non cohesive crack part appears. We discuss also the influence of all the parameters of the problem, in particular the non-uniform stress and cohesive model formulations, and study the sensitivity to imperfections.
\end{abstract}

Keywords: cohesive zone model, stress gradient, crack evolution.

\section{INTRODUCTION}

Griffith's theory of fracture [1] is based on the concept of critical energy release rate $G_{c}$ which comes from the fundamental but somewhat too restrictive assumption that the surface energy associated with a crack is proportional to the area of the crack (at least in a homogeneous and isotropic body), or, equivalently, that there is no interaction between the lips of a crack. It remains the most used approach in fracture mechanics thanks to its simplicity in terms of material behavior. However, this theory contains some major drawbacks. In particular, since Griffith's model does not contain a critical stress, (i) it allows stress singularity and (ii) cannot give an account of the nucleation of crack in a sound body. Accordingly, cohesive-force models have been introduced with main goal to prohibit these types of unphysical singularities by allowing finite stresses only.

(C) 2018 Vietnam Academy of Science and Technology 
Specifically, following the ideas of [2] and [3], many such models have been proposed and tested, see for instance [4-9].

Barenblatt's model radically differs from Griffith's model as far as the nucleation of cracks is concerned. Indeed, it turns out that the cohesive models, because they contain a critical stress, are also able to explain the process of nucleation of a crack in a sound body whereas Griffith's model cannot, in general. The first author who was able to establish such a result in a rigorous manner was [10] in a one-dimensional setting by using a variational approach and introducing a stability criterion. With such a criterion, a complete comparison between the two types of models can be carried out in the 1D restricted setting, see [11]. Specifically, assuming that the surface energy density which governs the cohesive forces is a smooth increasing concave function $\Phi[u]]$ of the displacement jump $[[u]$, then the stability criterion requires that the stress field $\sigma(x)$ at equilibrium be everywhere less than the derivative at 0 of the energy density function, say $\sigma(x) \leq \sigma_{c}:=\Phi^{\prime}(0)$. Therefore, $\sigma_{c}$ plays the role of the material critical stress. This result can be extended to a general 3D setting, still by using the stability criterion. In particular, assuming that the material is isotropic and hence that the surface energy density is only a function of the normal displacement jump and of the norm of the tangential displacement jump across the lips of the crack, say $\Phi(\llbracket[\mathbf{u}] \cdot \mathbf{n}, \|[[\mathbf{u}]]-(\llbracket \mathbf{u}] \cdot \mathbf{n}) \mathbf{n} \|)$ where $\mathbf{n}$ denotes the local unit normal vector to the crack, it is stated in [12] and proved in [13] that the criterion of nucleation of a crack takes the form of an intrinsic curve in the Mohr stress plane which involves the directional derivatives at $(0,0)$ of $\Phi$. However, this result only says that a cohesive crack will appear somewhere in the body when the stress field predicted by a pure elastic response reaches a threshold, but it says nothing on the growth process of these nucleated cracks. To treat this delicate issue, one must include in a unique formulation both the nucleation and the propagation of (cohesive) cracks. In essence, that is one of the main purposes of the variational approach to fracture, see [14] for an overview. In this context of cohesive force models, some partial results have already been obtained. For instance, $[15,16]$ study the size and shape effects of preexisting defects in the case of Dugdale's model. It is in particular shown that the value of the loading at which the first cohesive crack occurs strongly depends on the shape of the preexisting defect. On the other hand, sufficiently small defects have practically no influence on the overall resistance of a structure.

Besides these first results on shape or size effects, $[17,18]$ treat the problem of the nucleation and the propagation of a cohesive crack at the tip of a notch, still in the context of Dugdale's model. In such a situation, since the notch would induce a singularity of the stress if the response was purely elastic, there exists no elastic phase in the loading process, a cohesive crack is created at the tip of the notch as soon as a load is applied. The length of the crack and its opening grow in such a manner that there is no singularity of the stresses. During a first stage of the loading, the growth of the crack is stable, but it becomes unstable when the crack opening just at the notch tip reaches the critical value $\delta_{c}$ associated with Dugdale's model. Then a macroscopic crack is created by instability, the length of which being governed by a condition of energy conservation. In the case where the material characteristic length is small by comparison to the overall dimension 
of the body, it is even possible by using a two-scale technique to obtain in a closed form the formula giving the load at which a macroscopic appears at the notch tip.

The goal of the present work is to consider the same problem as in $[17,18]$ but for another type of structure. Specifically, we assume that the body contains neither a notch nor any corner which would induce elastic singularities. In other words the stress field associated with a pure elastic response is assumed to be smooth and bounded, but non uni- form. Considering a symmetric structure submitted to an increasing loading and adopting Dugdale's law as the cohesive force model, a cohesive crack appears at a material point where the normal stress is maximal when the loading reaches a critical value. The question is then to study the process of propagation of this crack and to highlight the stabilizing effects of the stress gradients. Specifically, one shows that the growth of the crack is first progressive, which means that it depends continuously on the loading parameter, by virtue of the non-uniformity of the stress field. In fact, the first stage of the crack growth is controlled by the second derivatives of the stress field. In a second times, when the loading reaches a value such that the crack opening at its center reaches the critical value $\delta_{c}$, then a non cohesive zone appears in the center and the propagation becomes brutal, the size of the crack jumping instantaneously to a value which is fixed by the characteristic length of the stress gradient. This second critical value of the loading can be seen as the moment where a genuinely macroscopic and non cohesive crack appears. The main feature of the paper is to obtain all the results in a closed form by using the method of complex potentials and a two-scale technique.

\section{SETTING OF THE PROBLEM AND MAIN ASSUMPTIONS}

\subsection{The body, its elastic behavior and its loading}

Throughout the paper the analysis is made in a plane strain setting. One uses a Cartesian system $\left(x_{1}, x_{2}, x_{3}\right)$ with its canonical orthonormal basis $\left(\mathbf{e}_{1}, \mathbf{e}_{2}, \mathbf{e}_{3}\right)$. The reference configuration of the body is the open subset $\Omega$ of $\mathbb{R}^{2}$ in the plane $\left(x_{1}, x_{2}\right)$ (Fig. 1 ). The body is made of an isotropic brittle material whose elastic behavior before cracking is characterized by its Lamé coefficients $\lambda$ and $\mu$ (or equivalently by its Young modulus $E$ and its Poisson ratio $v$ ). This material can be damaged by cracks the behavior of which is governed by the Dugdale model (see below for a precise statement of that model). The body is submitted to a proportional loading parameterized by the increasing parameter $t>0$ called from now the time. Accordingly, if the response were purely elastic, then the displacement field $\mathbf{u}^{\mathrm{e} 1}(t)$ and the stress fields $\sigma^{\mathrm{e} 1}(t)$ at time $t$ would be the solutions of the following linear boundary value problem

$$
\begin{cases}\operatorname{div} \sigma^{\mathrm{el}}(t)=0 & \text { in } \Omega \\ \sigma^{\mathrm{el}}(t)=\lambda \operatorname{tr}\left(\varepsilon\left(\mathbf{u}^{\mathrm{el}}(t)\right)\right) \mathbf{I}+2 \mu \varepsilon\left(\mathbf{u}^{\mathrm{el}}(t)\right) & \text { in } \Omega \\ \sigma^{\mathrm{el}}(t) \mathbf{n}=t \mathbf{F} & \text { on } \partial_{N} \Omega \\ \mathbf{u}^{\mathrm{el}}(t)=t \mathbf{U} & \text { on } \partial_{D} \Omega\end{cases}
$$

In (1), $\varepsilon\left(\mathbf{u}^{\mathrm{e} 1}(\mathrm{t})\right)$ denotes the strain field associated with the displacement field $\mathbf{u}^{\mathrm{e}}(t)$, i.e. the symmetric part of the gradient of $\mathbf{u}^{\mathrm{e}}(t)$. The body forces are neglected, $\partial_{N} \Omega$ 


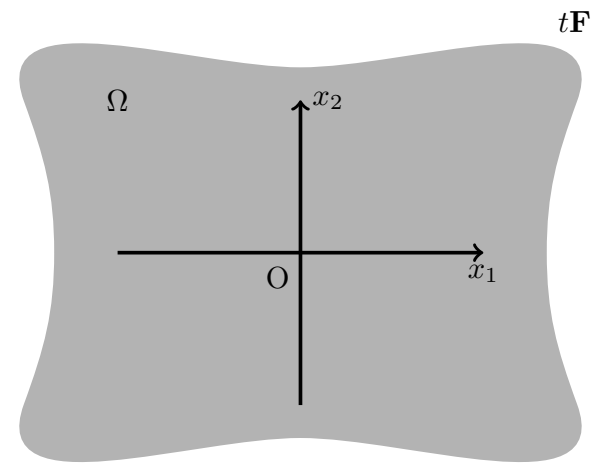

Fig. 1. The body and its loading

represents the part of the boundary where the surface forces are (progressively) applied whereas $\partial_{D} \Omega$ represents the complementary part of the boundary where the displacements are prescribed. The loading is proportional in the sense that the intensity of the applied forces and the amplitude of the prescribed displacements are proportional to the parameter $t$. By virtue of the linearity of the problem (1), its solution depends linearly on $t$ and hence can be written

$$
\mathbf{u}^{\mathrm{el}}(t)=t \mathbf{u}^{\mathrm{el}}, \quad \sigma^{\mathrm{el}}(t)=t \sigma^{\mathrm{el}}
$$

where $\left(\mathbf{u}^{\mathrm{e} 1}, \sigma^{\mathrm{e} 1}\right)$ are solutions of the following linear elastic problem

$$
\begin{cases}\operatorname{div} \sigma^{\mathrm{el}}=0 & \text { in } \Omega \\ \sigma^{\mathrm{el}}=\lambda \operatorname{tr}\left(\varepsilon\left(\mathbf{u}^{\mathrm{el}}\right)\right) \mathbf{I}+2 \mu \varepsilon\left(\mathbf{u}^{\mathrm{el}}\right) & \text { in } \Omega \\ \sigma^{\mathrm{el}} \mathbf{n}=\mathbf{F} & \text { on } \partial_{N} \Omega \\ \mathbf{u}^{\mathrm{el}}=\mathbf{U} & \text { on } \partial_{D} \Omega\end{cases}
$$

\subsection{Symmetry and smoothness assumptions}

We assume that the body is symmetric with respect to the two axes $x_{1}=0$ and $x_{2}=0$. Moreover, the loading preserves this symmetry and the elastic response enjoys the following properties:

(1) The shear stress $\sigma_{12}^{\mathrm{e} 1}$ vanishes on the axes $x_{1}=0$ and $x_{2}=0$. Consequently, the stress tensor is diagonal in the basis $\left(\mathbf{e}_{1}, \mathbf{e}_{2}\right)$ at each point of the axes and its eigenvalues are respectively denoted $\sigma_{1}^{\mathrm{e} 1}$ and $\sigma_{2}^{\mathrm{e} 1}$;

(2) The elastic stress field $\sigma^{\mathrm{e} 1}(\mathbf{x})$ is a smooth function of $\mathbf{x}$. The maximum of $\sigma_{n n}^{\mathrm{e} 1}(\mathbf{x})$ is reached at the origin $\mathbf{O}=(0,0)$, in the direction $\mathbf{n}=\mathbf{e}_{2}$ and is positive.

These symmetry and smoothness assumptions on the elastic stress field induce some properties on the repartition of the stresses along the axis $x_{2}=0$ that will be useful in the sequel. Specifically,

(1) The stress vector $\sigma^{\mathrm{e} 1}\left(x_{1}, 0\right) \mathbf{e}_{2}$ is purely normal, say

$$
\sigma^{\mathrm{el}}\left(x_{1}, 0\right) \mathbf{e}_{2}=\Sigma\left(x_{1}\right) \mathbf{e}_{2} \text {. }
$$


(2) The normal stress distribution $\Sigma\left(x_{1}\right)$ is an even smooth function of $x_{1}$ which is maximum at $x_{1}=0$. Introducing the characteristic length $\ell$ of the stress gradient,

$$
\ell:=2 \sqrt{\frac{\sigma_{2}^{\mathrm{el}}(0,0)}{-\sigma_{2,11}^{\mathrm{el}}(0,0)}},
$$

and expanding the normal stress distribution $\Sigma\left(x_{1}\right)$ in the expression (4) with respect to $x_{1}$, near $x_{1}=0$, up to the infinity order, we obtain

$$
\Sigma\left(x_{1}\right)=\sigma_{2}^{\mathrm{el}}(0,0)\left(1-\sum_{n=1}^{\infty} \alpha_{n} \frac{x_{1}^{2 n}}{\ell^{2 n}}\right),
$$

where $\sigma_{2}^{\mathrm{e} 1}(0,0)$ represents the normal stress at the origin and the coefficients $\left(\alpha_{n}\right)$ characterize the non-uniformity of stress field in the neighborhood of the point $\mathrm{O}=(0,0)$. By definition of $\ell, \alpha_{1}=2$.

\subsection{Dugdale's model of crack opening}

The nucleation and the growth of cracks in the body are governed by Dugdale's cohesive force model whose main ingredients are recalled here. This model, formulated in energetic terms, is based on the fundamental assumption that the surface energy density $\Phi$ depends in a non trivial manner on the displacement jump, unlike the Griffith model in which $\Phi$ is assumed to be constant. So in Dugdale's model, by assuming that the crack is always in mode I, i.e. that only the normal displacement is discontinuous, the surface energy density reads:

$$
\left.\Phi\left(\llbracket\left[u_{n}\right]\right]\right)=\left\{\begin{array}{lll}
+\infty & \text { if } & \llbracket u_{n} \rrbracket<0 \\
G_{c} \llbracket\left[u_{n} \rrbracket / \delta_{c}\right. & \text { if } 0 \leq \llbracket\left[u_{n}\right] \leq \delta_{c} \\
G_{c} & \text { if } \llbracket\left[u_{n} \rrbracket \geq \delta_{c}\right.
\end{array}\right.
$$

In (7), $\left[\left[u_{n}\right]\right]$ denotes the normal displacement jump, $G_{c}$ is the critical energy release rate of Griffth's theory, whereas $\delta_{c}$ is an internal length characteristic of the cohesive forces model. The ratio $G_{c} / \delta_{c}$ has the dimension of a stress, say $\sigma_{c}$

$$
\sigma_{c}:=\frac{G_{c}}{\delta_{c}}
$$

In terms of the cohesive forces, the normal stress $\sigma_{n n}$ giving the interaction between the crack lips is equal to $\sigma_{c}$ as long as $0<\left[\left[u_{n}\right]\right]<\delta_{c}$ and vanishes as soon as $\left[u_{n} \rrbracket>\delta_{c}\right.$,

$$
\sigma_{n n}\left\{\begin{array}{lll}
\leq \sigma_{c} & \text { if } & {\left[u_{n} \rrbracket=0\right.} \\
=\sigma_{c} & \text { if } & 0<\llbracket\left[u_{n}\right] \leq \delta_{c} \\
=0 & \text { if } & {\left[u_{n} \rrbracket\right]>\delta_{c}}
\end{array}\right.
$$

Therefore, the crack lips are generally divided into two zones: the so-called cohesive zone in which the cohesive forces are equal to $\sigma_{c}$ and a so-called non cohesive zone in which there are no cohesive forces. The evolution of Dugdale's surface energy density and normal stress in function of the normal displacement jump are shown in Fig. 2. 

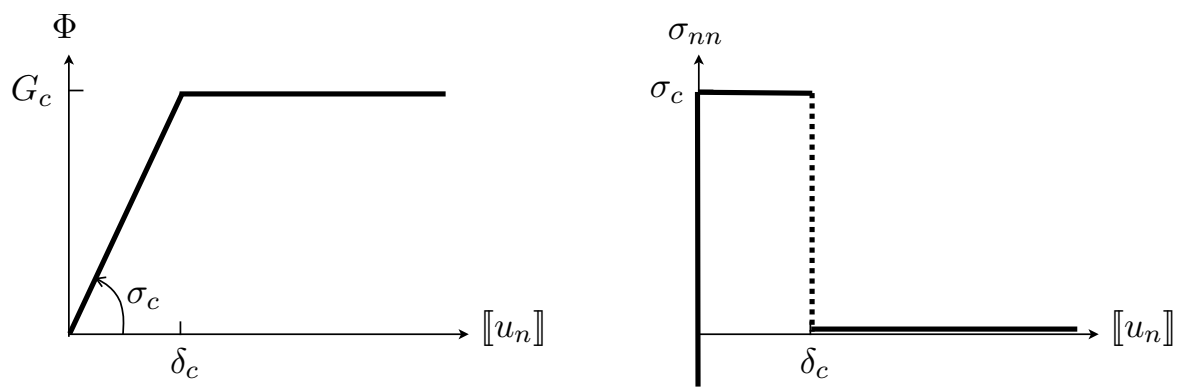

Fig. 2. Dugdale's surface energy density and normal stress in function of the normal displacement jump

Remark 1. The length $\delta_{c}$ characterizes the critical crack opening from which no more cohesive forces exist in Dugdale's model. It is a material characteristic length. But, in the plane strain calculations, another material characteristic length appears which involves also the elastic properties of the material. This length is defined by

$$
d_{c}=\frac{\pi}{8\left(1-v^{2}\right)} \frac{E}{\sigma_{c}} \delta_{c}
$$

and gives the order of magnitude of the length of the cohesive zone. In practice, since $E$ is much greater than $\sigma_{c}$ for usual materials, $d_{c}$ is much greater than $\delta_{c}$.

\subsection{Reduction of the problem with the help of the symmetry assumptions}

The evolution problem can be reduced with the help of the symmetry assumptions introduced in Subsection 2.2. Let us note however that, since the uniqueness of the solution is not ensured, the search for a solution respecting these symmetries constitutes additional assumptions. The first extra assumption is the following

Hypothesis 1 (Centered crack path). At every time $t>0$, the jump set $S_{\mathbf{u}(t)}$ is either empty or an interval centered at $(0,0)$, i.e. there exists $a(t) \geq 0$ such that $S_{\mathbf{u}(t)}=(-a(t), a(t)) \times\{0\}$.

The second assumptions concerns the monotonicity of the opening of the crack lips.

Hypothesis 2 (Symmetry and monotonicity of $\left.\left[u(t)_{2}\right]\right]$ ). When the jump set $S_{\mathbf{u}(t)}$ is not empty, the opening $\left[u(t)_{2}\right](x)$ is an even continuous function of $x_{1}$, maximal at $x_{1}=0$ and decreasing to 0 when $\left|x_{1}\right|$ grows to $a(t)$.

This second assumption limits the number of possibilities for the crack state at a given $t$. Specifically, we can distinguish the three following cases, see also Fig. 3:

(1) There exists no crack, i.e. $a(t)=0$ and $S_{\mathbf{u}(t)}=\varnothing$. The response is purely elastic and the set of all crack states of this type which satisfy the first order stability conditions is called the elastic branch;

(2) A crack exists but its opening at $x_{1}=0$ is less than the critical value $\delta_{c}$ associated with Dugdale's model, i.e. $a(t)>0$ and $\left.\llbracket u(t)_{2}\right](0) \leq \delta_{c}$. That corresponds to the case where the entire crack lips are submitted to the cohesive force $\sigma_{c}$. The 
set of all crack states of this type which satisfy the first order stability conditions is called the fully cohesive branch;

(3) A crack exists and its opening at $x_{1}=0$ is greater than the critical value $\delta_{c}$, i.e. $a(t)>0$ and $\left[u(t)_{2}\right](0)>\delta_{c}$. In that case, since the opening is a monotonic function of $\left|x_{1}\right|$ decreasing to 0 , there exists two symmetrical points $( \pm b(t), 0)$ with $0<b(t)<a(t)$ where the opening is equal to $\delta_{c}$. Therefore, by virtue of Dugdale's model, the crack is divided into two parts:

(a) The cohesive zone where the cohesive forces are equal to $\sigma_{c}$;

(b) The non cohesive zone where the cohesive forces vanish.

Specifically, that corresponds to the case where $a(t)>b(t)>0$ and

$$
\left\{\begin{array}{lll}
\text { if }\left|x_{1}\right|<b(t), & \text { then } & {\left[u(t)_{2}\right]\left(x_{1}\right)>\delta_{c}, \quad \sigma(t)_{22}\left(x_{1}, 0\right)=0} \\
\text { if } b(t)<\left|x_{1}\right|<a(t), & \text { then } \left.\left.\quad 0<\llbracket u(t)_{2}\right]\right]\left(x_{1}\right)<\delta_{c}, \quad \sigma(t)_{22}\left(x_{1}, 0\right)=\sigma_{c}
\end{array}\right.
$$

The set of all crack states of this type which satisfy the first order stability conditions is called the partially non cohesive branch.
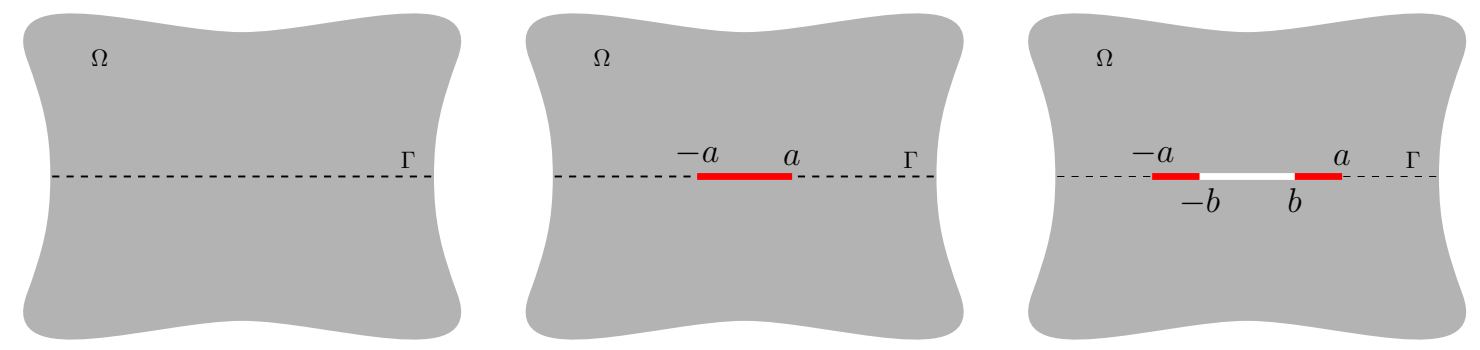

Fig. 3. The three possible types of crack state: (i) no crack, (ii) fully cohesive crack, (iii) partially non cohesive crack

The positions $a(t)$ and $b(t)$ of the tips of the cohesive zone and non cohesive zone, when they exist, have to be determined. The absence of singularity at the non cohesive zone tips and the continuity of the crack opening at the cohesive zone tips give us the conditions for $a(t)$ and $b(t)$, as shown in the following Propositions

Proposition 2.1 (Vanishing of the stress intensity factor $K_{I}$ at the tips of the cohesive crack). Since the normal stress $\sigma(t)_{22}$ must be bounded everywhere on $\Gamma$, no singularity can exist at the tips of the cohesive crack and hence the stress intensity factor $\mathrm{K}_{\mathrm{I}}$ must vanish at $x_{1}= \pm a(t)$.

Proposition 2.2 (Critical opening at the non cohesive crack tip). The position $b(t)$ of the non cohesive crack tips, when they exist, must be such that the opening at these tips be equal to $\delta_{c}$

$$
\left.\llbracket u(t)_{2}\right]( \pm b(t))=\delta_{c} .
$$




\section{DUGDALE'S COHESIVE MODEL, GENERALISED STRESS DISTRIBUTION}

\subsection{Analytical calculation with a two-scale approach}

We construct now a solution of the crack evolution problem in a closed form, but under the condition that the material characteristic length $d_{c}$ defined in (10) is small by comparison with the characteristic length $L$ of the body. Moreover, we assume that the stress field is genuinely non uniform by considering that $\ell$ is of the same order as or much smaller than $L$

$$
d_{c} \ll L, \quad \ell \lesssim L
$$

This hierarchy of the lengths allows us to use a two-scale approach to construct the solution (which will be hence an approximate solution only). The construction follows the procedure described in the previous subsection.

\subsubsection{Determination of the elastic branch}

Once the original elastic problem (3) is solved, one obtains the normal stress distribution $\sigma_{22}^{\mathrm{e} 1}\left(x_{1}\right)=\Sigma\left(x_{1}\right)$ along the axis $\Gamma$. Therefore, the coefficients $\left(\alpha_{n}\right)$ of the series (6) can be obtained. In addition, the critical time $t_{e}$ which corresponds to the the nucleation of cohesive crack when the maximal tensile stress associated to elastic response reaches the critical stress $\sigma_{c}$ can be calculated as

$$
t_{e}:=\frac{\sigma_{c}}{\sigma_{22}^{\mathrm{el}}(0)} .
$$

\subsubsection{Determination of the fully cohesive branch}

One considers the case of a fully cohesive crack with a length $2 a>0$ at time $t>0$. For given $a$ and $t$, we define the associated displacement and stress fields as the unique solution, denoted $(\mathbf{u}[t, a], \sigma[t, a])$, of the following linear elastic problem posed on the cracked body with uniform cohesive forces on the crack lips

$$
\left\{\begin{array}{lll}
\operatorname{div} \sigma[t, a]=0 & \text { in } \Omega \backslash([-a, a] \times\{0\}) \\
\sigma[t, a]=\lambda \operatorname{tr}(\varepsilon(\mathbf{u}[t, a])) \mathbf{I}+2 \mu \varepsilon(\mathbf{u}[t, a]) & \text { in } \Omega \backslash([-a, a] \times\{0\}) \\
\mathbf{u}[t, a]=t \mathbf{U} & \text { on } \partial_{D} \Omega \\
\sigma[t, a] \mathbf{n}=t \mathbf{F} & \text { on } \partial_{N} \Omega \\
\sigma[t, a] \mathbf{e}_{2}=\sigma_{c} \mathbf{e}_{2} & \text { on }[-a, a] \times\{0\}
\end{array}\right.
$$

Since the crack length is small, the crack should perturb the elastic fields only in a neighborhood of the origin. Therefore, if we introduce in (13) the gaps of the solution with the elastic fields, i.e.

$$
\overline{\mathbf{u}}[t, a]=\mathbf{u}[t, a]-t \mathbf{u}^{\mathrm{el}}, \quad \bar{\sigma}[t, a]=\sigma[t, a]-t \sigma^{\mathrm{el}},
$$

then $\bar{\sigma}[t, a](\mathbf{x})$ should tend to 0 when $\|\mathbf{x}\|$ becomes large by comparison with $a$. Moreover, on the lips of the crack the gap of the normal stress verifies $\bar{\sigma}[t, a]_{22}\left(x_{1}\right)=\sigma_{c}-t \Sigma\left(x_{1}\right)$ where $\Sigma\left(x_{1}\right)$ is given by (6). Accordingly, using (12) leads to

$$
\bar{\sigma}[t, a]_{22}=\left(1-\frac{t}{t_{e}}\right) \sigma_{c}+\frac{t}{t_{e}} \sigma_{c} \sum_{n=1}^{\infty} \alpha_{n} \frac{x_{1}^{2 n}}{\ell^{2 n}} .
$$


These considerations allow us to write the problem giving the gaps in the neighborhood of the origin as follows

$$
\begin{cases}\operatorname{div} \bar{\sigma}[t, a]=0 & \text { in } \mathbb{R}^{2} \backslash([-a, a] \times\{0\}) \\ \bar{\sigma}[t, a]=\lambda \operatorname{tr}(\varepsilon[t, a]) \mathbf{I}+2 \mu \varepsilon(\overline{\mathbf{u}}[t, a]) & \text { in } \mathbb{R}^{2} \backslash([-a, a] \times\{0\}) \\ \bar{\sigma}[t, a] \rightarrow 0 & \text { when }|\mathbf{x}| \rightarrow \infty \\ \bar{\sigma}[t, a]\left(x_{1}, 0\right) \mathbf{e}_{2}=\sigma_{c}\left(1-\frac{t}{t_{e}}+\frac{t}{t_{e}} \sum_{n=1}^{\infty} \alpha_{n} \frac{x_{1}^{2 n}}{\ell^{2 n}}\right) \mathbf{e}_{2} & \text { when } \quad x_{1} \in(-a, a)\end{cases}
$$

This problem is a particular case of a family of plane elastic problems which can be solved with the method of complex potentials developed by [15]. Without detailing the calculation steps, we can directly use the results by identifying the normal stress distribution with

$$
\mathrm{T}\left(x_{1}\right)=\sigma_{c}\left(1-\frac{t}{t_{e}}+\frac{t}{t_{e}} \sum_{n=1}^{\infty} \alpha_{n} \frac{x_{1}^{2 n}}{\ell^{2 n}}\right) .
$$

After the calculation of the integral, the stress intensity factor $\mathrm{K}_{\mathrm{I}}[t, a]$ is given by

$$
\mathrm{K}_{\mathrm{I}}[t, a]=\sigma_{c} \sqrt{\pi a}\left[\frac{t}{t_{e}}-1-\frac{t}{t_{e}} \sum_{n=1}^{\infty}\left(\frac{\alpha_{n}}{\ell^{2 n}} a^{2 n} \prod_{i=1}^{n} \frac{2 i-1}{2 i}\right)\right] .
$$

The condition $\mathrm{K}_{\mathrm{I}}[t, a]=0$ gives an implicit relationship between the position of the non cohesive zone tips $a$ and the loading parameter $t$

$$
1-\frac{t_{e}}{t}=\sum_{n=1}^{\infty}\left(\frac{a^{2 n}}{\ell^{2 n}} \alpha_{n} \prod_{i=1}^{n} \frac{2 i-1}{2 i}\right) .
$$

This solution is valid as long as the crack opening at $x_{1}=0$ remains less than $\delta_{c}$, and provides that the normal stress is less that $\sigma_{c}$ all along the axis. The complex analysis method allows us to investigate the crack opening. Indeed, by using the normal stress distribution (16) the derivative of the complex potential $\varphi^{\prime}(z)$ can be expressed as follows

$$
\begin{aligned}
\varphi^{\prime}(z)= & -\frac{\sigma_{c}}{2}\left(\frac{t}{t_{e}}-1\right)+\frac{\sigma_{2}}{2} \frac{t}{t_{e}} \sum_{n=1}^{\infty} \alpha_{n} \frac{z^{2 n}}{\ell^{2 n}} \\
& i \frac{\sigma_{c}}{2} \frac{t}{t_{e}} z \sqrt{a(t)^{2}-z^{2}} \sum_{n=1}^{\infty} \frac{\alpha_{n}}{\ell^{2 n}}\left[z^{2(n-1)}+\sum_{i=1}^{n-1} \frac{(2 i-1) ! !}{(2 i) ! !} a(t)^{2 i} z^{2(n-i-1)}\right],
\end{aligned}
$$

where the crack length $a(t)$ is given by (18). In this expression, we denote

$$
(2 i) ! !:=\prod_{j=1}^{i} 2 j, \quad(2 i-1) ! !:=\prod_{j=1}^{i}(2 j-1) .
$$

Let us now determine the crack opening. Let $z^{ \pm}=x_{1} \pm i 0$ be the points on the lips of the crack at $x_{1},\left|x_{1}\right| \leq a(t)$. By using the conventional notations $0 !=(-1) !=1$, we deduce 
the complex potential jump as follows

$$
\llbracket \varphi \rrbracket^{\prime}\left(x_{1}\right)=-i \sigma_{c} x_{1} \sqrt{a(t)^{2}-x_{1}^{2}} \frac{t}{t_{e}} \sum_{n=1}^{\infty}\left(\frac{\alpha_{n}}{\ell^{2 n}} \sum_{i=0}^{n-1} \frac{(2 i-1) ! !}{(2 i) ! !} a(t)^{2 i} x_{1}^{2(n-i-1)}\right) .
$$

By a straightforward integration and using the fact that $\llbracket \varphi \rrbracket( \pm a(t))=0$, one gets $\llbracket \varphi \rrbracket\left(x_{1}\right)$. Besides, we have the following relationship

$$
\llbracket\left[u_{2} \rrbracket\left(x_{1}\right)=\frac{4\left(1-v^{2}\right)}{i E} \llbracket \varphi \rrbracket\left(x_{1}\right) .\right.
$$

Using the expression of the characteristic length of material $d_{c}$ given by (10), the crack opening at the origin reads as

$$
\left[u(t)_{2}\right](0)=\frac{\pi \delta_{c}}{2 d_{c}} \frac{t}{t_{e}} \sum_{n=1}^{\infty} \frac{\alpha_{n}}{\ell^{2 n}} a(t)^{2 n+1} \sum_{i=0}^{n-1} \frac{(2 i-1) ! !(2(n-i-1)) ! !}{(2 i) ! !(2(n-i)+1) ! !} .
$$

Since crack length is an increasing function of the loading parameter $t$, the crack opening at the origin $x_{1}=0$ is also an increasing function of $t$ for $t \geq t_{e}$. When $\left.\llbracket u(t)_{2}\right](0)$ reaches the critical value $\delta_{c}$, the fully cohesive branch finishes and a non cohesive zone will appear at the center of crack. Le critical loading parameter $t_{i}$ corresponding to this instant can be calculated thanks to the following implicit function

$$
\frac{t}{t_{e}} \sum_{n=1}^{\infty} \frac{\alpha_{n}}{\ell^{2 n}} a(t)^{2 n+1} \sum_{i=0}^{n-1} \frac{(2 i-1) ! !(2(n-i-1)) ! !}{(2 i) ! !(2(n-i)+1) ! !}=\frac{2}{\pi} d_{c} .
$$

\subsubsection{Determination of the partially non cohesive branch}

Let us consider now the partially non cohesive crack evolution at time $t$ whose non cohesive length is $2 b$ and the tips of the cohesive zones are at $\pm a$. For given $(a, b, t)$ with $0<b<a$ and $t>0$, we define the associated displacement and stress fields as the unique solution, denoted $(\mathbf{u}[t, a, b], \sigma[t, a, b]$, of the following linear elastic problem posed on the cracked body with non uniform cohesive forces on the crack lips

$$
\begin{cases}\operatorname{div} \sigma[t, a, b]=0 & \text { in } \Omega \backslash([-a, a] \times\{0\}) \\ \sigma[t, a, b]=\lambda \operatorname{tr}(\varepsilon(\mathbf{u}[t, a, b])) \mathbf{I}+2 \mu \varepsilon(\mathbf{u}[t, a, b]) & \text { in } \Omega \backslash([-a, a] \times\{0\}) \\ \mathbf{u}[t, a, b]=t \mathbf{U} & \text { on } \partial_{D} \Omega \\ \sigma[t, a, b] \mathbf{n}=t \mathbf{F} & \text { on } \partial_{N} \Omega \\ \sigma[t, a, b] \mathbf{e}_{2}=\mathbf{0} & \text { on }(-b, b) \times\{0\} \\ \sigma[t, a, b] \mathbf{e}_{2}=\sigma_{c} \mathbf{e}_{2} & \text { on }((-a,-b) \cup(b, a)) \times\{0\}\end{cases}
$$

Since $0<b<a \ll L$, the crack should perturb the elastic fields only in a neighborhood of the origin. Consequently, one can introduce in (24) the gaps of solution with elastic fields, i.e.

$$
\overline{\mathbf{u}}[t, a, b]=\mathbf{u}[t, a, b]-t \mathbf{u}^{\mathrm{el}}, \quad \bar{\sigma}[t, a, b]=\sigma[t, a, b]-t \sigma^{\mathrm{el}}
$$


Using the same approximations and hypothesis as the case of a fully cohesive crack, the local problem reads as follows

$$
\begin{cases}\operatorname{div} \bar{\sigma}[t, a, b]=0 & \text { in } \mathbb{R}^{2} \backslash([-a, a] \times\{0\}) \\ \bar{\sigma}[t, a, b]=\lambda \operatorname{tr}(\varepsilon(\overline{\mathbf{u}}[t, a, b])) \mathbf{I}+2 \mu \varepsilon(\overline{\mathbf{u}}[t, a, b]) & \text { in } \mathbb{R}^{2} \backslash([-a, a] \times\{0\}) \\ \bar{\sigma}[t, a, b](\mathbf{x}) \rightarrow 0 & \text { when }|\mathbf{x}| \rightarrow \infty \\ \bar{\sigma}[t, a, b]\left(x_{1}, 0\right) \mathbf{e}_{2}=\mathrm{T}\left(x_{1}\right) \mathbf{e}_{2} & \text { on } \quad(-a, a) \times\{0\}\end{cases}
$$

where

$$
\mathrm{T}\left(x_{1}\right)=\left\{\begin{array}{lll}
\left(-\frac{t}{t_{e}}+\frac{t}{t_{e}} \sum_{n=1}^{\infty} \alpha_{n} \frac{x_{1}^{2 n}}{\ell^{2 n}}\right) \sigma_{c} & \text { if } & \left|x_{1}\right|<b \\
\left(1-\frac{t}{t_{e}}+\frac{t}{t_{e}} \sum_{n=1}^{\infty} \alpha_{n} \frac{x_{1}^{2 n}}{\ell^{2 n}}\right) \sigma_{c} & \text { if } \quad b<\left|x_{1}\right|<a
\end{array}\right.
$$

The integral calculation give us the intensity factor $\mathrm{K}_{\mathrm{I}}[t, a, b]$ as follows

$$
\mathrm{K}_{\mathrm{I}}[t, a, b]=\sigma_{c} \sqrt{\pi a}\left[\frac{t}{t_{e}}-1-\frac{t}{t_{e}} \sum_{n=1}^{\infty}\left(\frac{a^{2 n}}{\ell^{2 n}} \alpha_{n} \prod_{i=1}^{n} \frac{2 i-1}{2 i}\right)+\frac{2}{\pi} \arcsin \frac{b}{a}\right]
$$

The condition $\mathrm{K}_{\mathrm{I}}[t, a, b]=0$ gives us the first relationship between the crack lengths $(a, b)$ and the loading parameter $t$

$$
\frac{t}{t_{e}}\left[1-\sum_{n=1}^{\infty}\left(\frac{a^{2 n}}{\ell^{2 n}} \alpha_{n} \prod_{i=1}^{n} \frac{2 i-1}{2 i}\right)\right]=\frac{2}{\pi} \arccos \frac{b}{a} .
$$

One can investigate the complex potential jump on cohesive zone, i.e. $\left[[\varphi]^{\prime}\left(x_{1}\right)\right.$ pour $b<$ $\left|x_{1}\right|<a$, to compute the crack opening $\left[u[t, a, b]_{2}\right]\left(x_{1}\right)$. Using normal stress distribution (26) and the relationship (28), we obtain

$$
\begin{aligned}
\llbracket \varphi]^{\prime}\left(x_{1}\right)= & {\left[\frac{1}{\pi} \operatorname{arctanh}\left(\frac{b}{x_{1}} \sqrt{\frac{a^{2}-x_{1}^{2}}{a^{2}-b^{2}}}\right)\right.} \\
& \left.+\frac{x_{1} \sqrt{a^{2}-x_{1}^{2}}}{2} \frac{t}{t_{e}} \sum_{n=1}^{\infty}\left(\frac{\alpha_{n}}{\ell^{2 n}} \sum_{i=0}^{n-1} \frac{(2 i-1) ! !}{(2 i) ! !} a^{2 i} x_{1}^{2(n-i-1)}\right)\right] .
\end{aligned}
$$

By a straightforward integration and using the fact that $\llbracket \varphi \rrbracket( \pm a(t))=0$, one gets $\llbracket \varphi \rrbracket\left(x_{1}\right)$. Using the relationship (21), one can deduce the crack opening at the cohesive zone tips $x_{1}= \pm b$ as follows

$$
\begin{aligned}
& \llbracket u[a, b, t]_{2} \rrbracket(b)=\frac{\pi \delta_{c}}{2 d_{c}}\left[2 \frac{b}{\pi} \ln \frac{a}{b}+\frac{t}{t_{e}} \sqrt{1-\frac{b^{2}}{a^{2}}} \sum_{n=1}^{\infty} \frac{\alpha_{n}}{\ell^{2 n}} a^{2 n+1} \sum_{i=0}^{n-1} \frac{(2 i-1) ! !(2(n-i-1)) ! !}{(2 i) ! !(2(n-i)+1) ! !}\right. \\
& +\frac{t}{t_{e}} \sqrt{1-\frac{b^{2}}{a^{2}}} \sum_{n=1}^{\infty} \frac{\alpha_{n}}{\ell^{2 n}} a^{2 n+1} \sum_{i=0}^{n-1} \frac{(2 i-1) ! !(2(n-i-1)) ! !}{(2 i) ! !(2(n-i)+1) ! !} \sum_{j=0}^{n-i-2} \frac{(2 j+1) ! !}{(2 j+2) ! !}\left(\frac{b}{a}\right)^{2 j+2} \\
& \left.-\frac{t}{t_{e}} \sqrt{1-\frac{b^{2}}{a^{2}}} \sum_{n=1}^{\infty} \frac{\alpha_{n}}{\ell^{2 n}} a^{2 n+1} \sum_{i=0}^{n-1} \frac{(2 i-1) ! !}{(2 i) ! !} \frac{1}{2(n-i)+1}\left(\frac{b}{a}\right)^{2(n-i)}\right] .
\end{aligned}
$$


Using the condition given in Proposition 2.2, we obtain the second relationship between $a, b$ and $t$

$$
\begin{aligned}
& \frac{2}{\pi} d_{c}=\frac{2}{\pi} b \ln \frac{a}{b}+\frac{t}{t_{e}} \sqrt{1-\frac{b^{2}}{a^{2}}} \sum_{n=1}^{\infty} \frac{\alpha_{n}}{\ell^{2 n}} a^{2 n+1} \sum_{i=0}^{n-1} \frac{(2 i-1) ! !(2(n-i-1)) ! !}{(2 i) ! !(2(n-i)+1) ! !} \\
& +\frac{t}{t_{e}} \sqrt{1-\frac{b^{2}}{a^{2}}} \sum_{n=1}^{\infty} \frac{\alpha_{n}}{\ell^{2 n}} a^{2 n+1} \sum_{i=0}^{n-1} \frac{(2 i-1) ! !\left(2(n-i-1) ! ! !{ }^{n-i-2}\right.}{(2 i) ! !(2(n-i)+1) ! !} \sum_{j=0}^{n-i-2} \frac{(2 j+1) ! !}{(2 j+2) ! !}\left(\frac{b}{a}\right)^{2 j+2} \\
& -\frac{t}{t_{e}} \sqrt{1-\frac{b^{2}}{a^{2}}} \sum_{n=1}^{\infty} \frac{\alpha_{n}}{\ell^{2 n}} a^{2 n+1} \sum_{i=0}^{n-1} \frac{(2 i-1) ! !}{(2 i) ! !} \frac{1}{2(n-i)+1}\left(\frac{b}{a}\right)^{2(n-i)} \cdot
\end{aligned}
$$

\subsection{Representation of the three branches in particular cases}

We present in the present work the case of the 4th order normal stress distribution. This case corresponds to $n_{\max }=2$ in (6). In consequence, two parameters $\left(\alpha_{1}, \alpha_{2}\right)$ and the length $\ell$ are introduced to characterize the non uniform normal stress $\Sigma\left(x_{1}\right)$. Evidently, $\left(\alpha_{1}, \alpha_{2}\right)$ depend on the elastic solution with properties of structure, limit conditions, etc. In order to investigate analytically the problem of crack evolution, we choose the following set of values for the two parameters

$$
\alpha_{1}=2, \quad \alpha_{2}=\frac{8}{3}
$$

The normal stress distribution $\Sigma\left(x_{1}\right)$ of elastic response given by (6) becomes

$$
\Sigma\left(x_{1}\right)=\sigma_{c} \frac{t}{t_{e}}\left(1-2 \frac{x_{1}^{2}}{\ell^{2}}-\frac{8}{3} \frac{x_{1}^{4}}{\ell^{4}}\right) .
$$

Recall that the elastic branch begins at $t=0$ and finishes when the loading parameter $t$ the critical value $t_{e}$. This branch corresponds to the segment line $\left[0, t_{e}\right] \times\{0\}$ in the $(t, a)$ diagram. Beyond the loading $t_{e}$, the crack nucleates and propagates in the structure. One considers now this evolution, which contains a fully cohesive branch and a partially non cohesive branch, corresponding to the distribution (33) of $\Sigma\left(x_{1}\right)$.

\subsubsection{Fully cohesive branch}

At the instant $t$, a cohesive crack of length $2 a$ is present in the structure. The condition for the stress intensity factor $\mathrm{K}_{\mathrm{I}}[t, \pm a]=0$ gives a relationship between the crack length and the loading parameter given by (18) which simply reads here as

$$
\frac{t}{t_{e}}\left(1-\frac{a^{2}}{\ell^{2}}-\frac{a^{4}}{\ell^{4}}\right)=1
$$

The loading parameter $t$ is an increasing function of the crack length. Consequently, this relationship is reversible and implies that the crack length is a continuous, monotonically increasing function of the loading. This solution is no more valid as soon as the crack opening reaches the critical value $\delta_{c}$. Corresponding to the normal stress distribution 
(33), the crack opening at the origin given by the general expression (22) becomes

$$
\left.\llbracket u[t, a]_{2}\right](0)=8\left(1-v^{2}\right) \frac{\sigma_{c}}{E}\left[\frac{1}{3} \frac{t}{3} \frac{a(t)^{3}}{\ell^{2}}+\frac{2}{5} \frac{t}{t_{e}} \frac{a(t)^{5}}{\ell^{4}}\right],
$$

where $a(t)$ is computed in (34). Accordingly, the crack opening at the origin $x_{1}=0$ is also a monotonically increasing function of $t$ for $t \geq t_{e}$, and reaches the critical cohesive value $\delta_{c}$ at the instant $t_{i}$ given by

$$
\frac{d_{c}}{\pi \ell}=\frac{t_{i}}{t_{e}}\left(\frac{1}{3}+\frac{2}{5} \frac{a\left(t_{i}\right)^{2}}{\ell^{2}}\right) \frac{a\left(t_{i}\right)^{3}}{\ell^{3}}
$$

\subsubsection{Partially non cohesive branch}

From the instant $t>0$, a partially non cohesive crack exists with non cohesive part length $2 b$ and total length $2 a$. The condition on the stress intensity factor $\mathrm{K}_{\mathrm{I}}[t, a, b]=0$ gives us the first relationship between two length $(a, b)$ and the loading parameter $t$

$$
\frac{t}{t_{e}}\left(1-\frac{a^{2}}{\ell^{2}}-\frac{a^{4}}{\ell^{4}}\right)=\frac{2}{\pi} \arccos \frac{b}{a}
$$

Corresponding to the normal stress distribution (33), the crack opening at the tip of non cohesive part $x_{1}=b$ is expressed as follows

$$
\begin{aligned}
{\left[u[t, a, b]_{2}\right](b)=8\left(1-v^{2}\right) \frac{\sigma_{c}}{\pi} } & {\left[\frac{b}{\pi} \ln \frac{a}{b}+\frac{1}{3} \frac{t}{3} \frac{\left(a^{2}-b^{2}\right)^{3 / 2}}{\ell^{2}}\right.} \\
+ & \left.\frac{t}{t_{e}} \frac{1}{\ell^{4}}\left(\frac{2 a^{2}}{3}\left(a^{2}-b^{2}\right)^{3 / 2}-\frac{4}{15}\left(a^{2}-b^{2}\right)^{5 / 2}\right)\right] .
\end{aligned}
$$

The condition $\left[u[t, a, b]_{2}\right](b)=\delta_{c}$ provides the second relationship between $(a, b, t)$

$$
b \ln \frac{a}{b}+\frac{\pi}{3} \frac{t}{t_{e}} \frac{\left(a^{2}-b^{2}\right)^{3 / 2}}{\ell^{2}}+\frac{t}{t_{e}} \frac{\pi}{\ell^{4}}\left(\frac{2 a^{2}}{3}\left(a^{2}-b^{2}\right)^{3 / 2}-\frac{4}{15}\left(a^{2}-b^{2}\right)^{5 / 2}\right)=d_{c} .
$$

Accordingly, the two lengths $(a, b)$ are related to the loading parameter $t$ by two relationships (37) and (39). In order to study the partially non cohesive crack evolution, one introduces the following dimensionless variable

$$
\alpha=\frac{b}{a} \in(0,1) .
$$

By injecting (37) and this variable in (39), we obtain

$$
-\left(1-\frac{a^{2}}{\ell^{2}}-\frac{a^{4}}{\ell^{4}}\right)\left(\frac{a}{\ell} \alpha \ln \alpha+\frac{d_{c}}{\ell}\right)+\frac{2 \arccos \alpha}{3}\left(1-\alpha^{2}\right)^{3 / 2}\left[\frac{a^{3}}{\ell^{3}}+2 \frac{a^{5}}{\ell^{5}}-\frac{4}{5}\left(1-\alpha^{2}\right) \frac{a^{5}}{\ell^{5}}\right]=0 .
$$

For given $\alpha \in(0,1),(40)$ is a $5^{\text {th }}$ order equation for $\bar{a}:=a / \ell$ which depends on the parameter $\epsilon:=d_{c} / \ell$. This equation admits a unique solution, say $\bar{a}^{\epsilon}(\alpha)$, whose dependence on $\alpha$ is non monotonic. Specially, $\bar{a}^{\epsilon}(\alpha)$ starts from $a_{i} / \ell=\sqrt{1-t_{e} / t_{i}}$ at $\alpha=0$, then is first decreasing up to $a_{m} / \ell$ before to be increasing and finally tends to the limit 
$a_{c}:=\ell \sqrt{\frac{\sqrt{5}-1}{2}}<\ell$ when $\alpha$ tends to 1 , also see Fig. 4. Besides, (37) allows us to write $t / t_{e}$ as a function of $\alpha$ with the parameter $\epsilon$

$$
\frac{t}{t_{e}}=\bar{t}^{\epsilon}(\alpha):=\frac{2 \arccos \alpha}{\pi\left(1-\bar{a}^{\epsilon}(\alpha)^{2}-\bar{a}^{\epsilon}(\alpha)^{4}\right)} .
$$
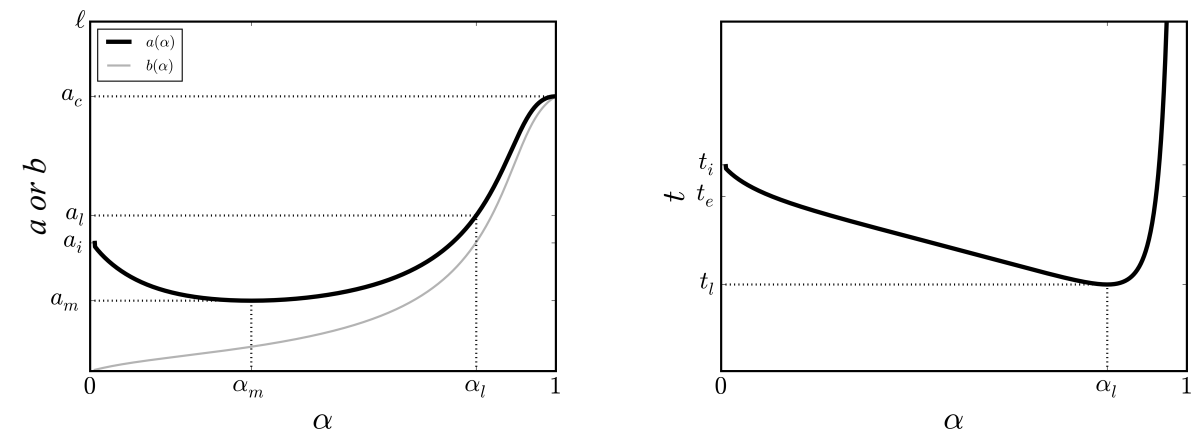

Fig. 4. Typical dependence of $a, b$ and $t$ on $\alpha=b / a$. Here the curves correspond to the case where $d_{c} / \ell=0.1$

The function $\bar{t}^{\epsilon}(\alpha)$ starts from $t_{i} / t_{e}$ at $\alpha=0$, and is first monotonically decreasing up to $t_{l} / t_{e}$, that minimum being reached at $\alpha=\alpha_{l}$. Then $\bar{t}^{\epsilon}(\alpha)$ is increasing to infinity when $\alpha$ tends to 1 , also see Fig. 4. Finally, the evolution of $b$ with $\alpha$ is given by

$$
\frac{b}{\ell}=\bar{b}^{\epsilon}(\alpha):=\alpha \bar{a}^{\epsilon}(\alpha) \text {. }
$$

Fig. 4 shows the evolution of $b$ with $\alpha$, which starts from 0 at $\alpha=0$ then increases monotonically and tends to $a_{c}=\ell \sqrt{\frac{\sqrt{5}-1}{2}}$ when $\alpha$ tends to 1 .

Accordingly, the triple $(t, a, b)$ satisfying (37) and (39) can be considered as two parametric curves $(t(\alpha), a(\alpha))$ and $(t(\alpha), b(\alpha))$ parameterized by $\alpha \in(0,1)$ and depending on the characteristic length $\ell$ and on the ratio $d_{c} / \ell$. In particular, the curve $(t(\alpha), a(\alpha))$ represents the partially non cohesive branch in the $(t, a)$ diagram, also see Figure 5 . Since the functions $\bar{a}^{\epsilon}(\alpha)$ and $\bar{t}^{\epsilon}(\alpha)$ are respectively non monotonic and monotonically decreasing for small $\alpha$, the partially non cohesive branch contains a snap-back in the neighborhood of $\left(t_{i}, a_{i}\right)$ and a limit point $\left(t_{l}, a_{l}\right)$, both points depending on $\ell$ and $d_{c}$. Accordingly, the branch has the shape of a loop which can be divided into two parts: the lower part between $\left(t_{i}, a_{i}\right)$ and $\left(t_{l}, a_{l}\right)$, the upper part after $\left(t_{l}, a_{l}\right)$.

Assuming that the critical stress $\sigma_{c}$ is fixed, one can study the dependence of the Dugdale's branches on $d_{c}$ at fixed $\ell$, or, on $\ell$ at fixed $d_{c}$. At fixed $\ell$ and for all $d_{c}$, the fully cohesive branch in the $(t, a)$ diagram is given by (34). Only the final point $\left(t_{i}, a_{i}\right)$ depends on $d_{c}$, and both $t_{i}$ and $a_{i}$ are increasing functions of $d_{c}$ (or $\left.\epsilon\right)$, also see Fig. 5 . On the one 
hand, when $d_{c}$ (or $\epsilon$ ) goes to 0 , then $t_{i}$ tends to $t_{e}$ and $a_{i} / \ell$ tends to 0 like $\epsilon^{1 / 3}$. On the other hand, when $d_{c} / \ell$ goes to infinity, then $a_{i}$ tends to $\ell$ and $t_{i}$ tends to infinity.

That means that the smaller the material length $d_{c}$, the weaker the stabilizing effect of the stress gradient. In the same manner, for the partially non cohesive branch, the smaller the material length $d_{c}$, the more accentuated the snap-back and the larger the size of the loop. At fixed $d_{c}$, for a given material, one can see the influence of the intensity of the stress gradient by comparing on Fig. 6 the Dugdale branches associated with different values of $\ell$. Let us recall that the higher the stress gradient, the smaller the length $\ell$, the case of a uniform stress field corresponding to $\ell=+\infty$. Accordingly, the higher the gradient, the greater the fully cohesive branch, the smaller the loop of

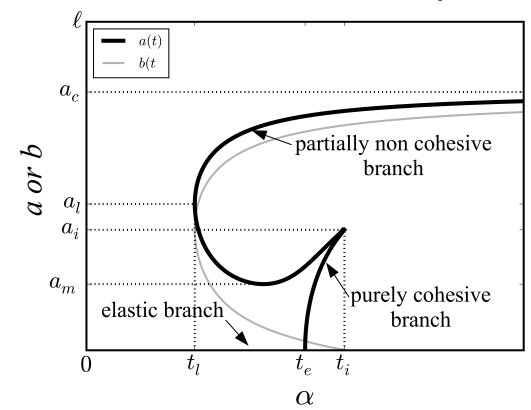

Fig. 5. Typical graphs of the three branches in the diagram $(t, a)$. The gray curve represents the evolution of the tip $b$ of the non cohesive crack for the partially non cohesive branch. Here the curves correspond to the case where $d_{c} / \ell=0.1$ the partially non cohesive branch and the smaller the final length of the crack.
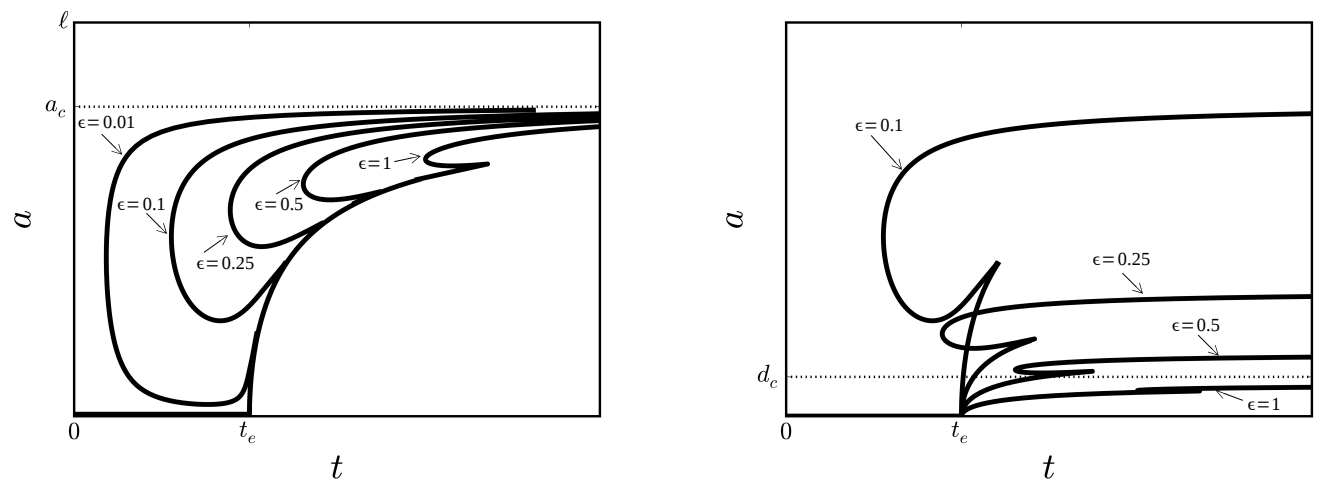

Fig. 6. Dependence of crack evolution branches on $d_{c}$ for fixed $\ell$, and on $\ell$ for fixed $d_{c}$

On the other hand, the response under monotonically increasing loading is shown in Fig. 7. Accordingly, the elastic solution est valid as long as the loading $t$ is in the interval $\left(0, t_{e}\right)$, then the fully cohesive crack nucleates and propagates continuously for $t \in\left(t_{e}, t_{i}\right)$. Finally, the crack length must jump at the instant $t_{i}$ corresponding to the apparition of non cohesive zone at the center of crack. If on neglects the inertial effects, at the instant $t_{i}$ the crack length jumps from the value $a_{i}$ to the value $a_{i}^{*}$ on the upper part of partially non cohesive branch.

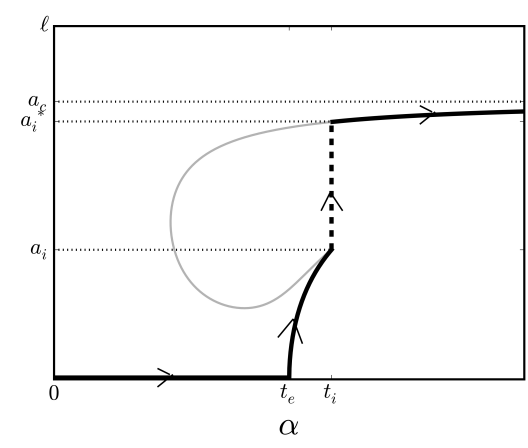

Fig. 7. Crack length evolution under monotonically increasing loading for $d_{c} / \ell=0.1$. Jump of crack length at $t=t_{i}$ 


\subsubsection{Sensibility to the imperfections}

It would seem that the shape of the loop and the snap-back part of the partially non cohesive branch do not play any role in the crack propagation under monotonic loading. However, the loop can be observed and even that it plays a fundamental role in presence of imperfections. Indeed, we consider the case where the imperfection corresponds to a preexisting non cohesive crack along the $x_{2}=0$, centered at $\mathbf{O}$ and of half-length $a_{0}<\ell$. Accordingly, the elastic response is no more regular, but the stress is singular at the tips $\pm a_{0}$ as soon as a loading is applied. There exists no more an elastic branch, but a cohesive zone must nucleate ahead the tips $\pm a_{0}$ as soon as $t>0$ with a length $a>a_{0}$ such that the singularity vanishes at the tips $\pm a$. Consequently, the relationship between $a, b$ and $t$ in order that the singularity vanishes remains given by (37). Besides, the crack opening at $x_{1}= \pm b$, namely $\left.\left[u[t, a, b]_{2}\right]\right](b)$, is always given by (38). Two relationships allows us to study the crack evolution with an initial imperfection under monotonically increasing loading. Specifically, the evolution can be divided into the two or three following parts, according to the value of $a_{0}$ :

(1) Cohesive phase: Growth of two symmetric purely cohesive zones, the non cohesive zone tips remaining at $\pm a_{0}$. The relationship between $a$ and $t$ is given by the condition $\mathrm{K}_{\mathbf{l}}\left[t, a, a_{0}\right]=0$ and hence (37) with $b=a_{0}$ leads to

$$
\frac{t}{t_{e}}=\frac{2}{\pi} \frac{\arccos \frac{a_{0}}{a}}{\left(1-\frac{a^{2}}{\ell^{2}}-\frac{a^{4}}{\ell^{4}}\right)} .
$$

In consequence, $a$ is a monotonically increasing function of $t$ starting from $a_{0}$ at $t=0$. That allows us to define the cohesive branch associated with the initial crack length $a_{0}$ in the diagram $(t, a)$. In addition, for $a$ et $t$ satisfying the relationship (43), the crack opening with $b=a_{0}$ expressed in (38) becomes

$$
\begin{aligned}
{\left[u\left[t, a, a_{0}\right]_{2}\right]\left(a_{0}\right)=} & 8\left(1-\mathbf{u}^{2}\right) \frac{\sigma_{c}}{E}\left[\frac{a_{0}}{\pi} \ln \frac{a}{a_{0}}+\frac{1}{3} \frac{t}{t_{e}} \frac{\left(a^{2}-a_{0}^{2}\right)^{3 / 2}}{\ell^{2}}\right. \\
& \left.+\frac{t}{t_{e}} \frac{1}{\ell^{4}}\left(\frac{2 a^{2}}{3}\left(a^{2}-a_{0}^{2}\right)^{3 / 2}-\frac{4}{15}\left(a^{2}-a_{0}^{2}\right)^{5 / 2}\right)\right] .
\end{aligned}
$$

This opening reaches the critical value $\delta_{c}$ when the triple $\left(a, a_{0}, t\right)$ satisfies the two conditions (37) and (39). Consequently, the triple is the point of the partially non cohesive branch of the perfect case which corresponds to $b=a_{0}$. The associated parameter $\alpha$ is given by the equation

$$
\bar{b}^{\epsilon}\left(\alpha_{0}\right) \ell=a_{0}
$$

its uniqueness being ensured by the monotonicity of the function $\bar{b}^{\epsilon}\left(\alpha_{0}\right)$. In other words the cohesive branch will finish when it intersects the loop of the perfect case. In conclusion, the cohesive branch starts from $\left(0, a_{0}\right)$ and finished at $\left(\bar{t}^{\epsilon}\left(\alpha_{0}\right) t_{e}, \bar{a}^{\epsilon}\left(\alpha_{0}\right) \ell\right)$.

(2) Possible jump of the crack length: Brutal propagation of the crack if the cohesive branch intersects the lower part of the loop of the perfect case. The intersection point between the purely cohesive branch and the loop of the perfect case depends on $a_{0}$. If $a_{0}$ is small enough, the intersection point is lower than the limit point of the loop, i.e. $\bar{a}^{\epsilon}\left(\alpha_{0}\right)<a_{l}$, 
the crack length must jump and the crack evolution is discontinuous after the purely cohesive branch. On the other hand, if $a_{0}$ is large enough, the intersection point is at or above the limit point of the loop, i.e. $\bar{a}^{\epsilon}\left(\alpha_{0}\right) \geq a_{l}$, the evolution can continuously follow that part of the curve in the sense of increasing time since the crack length increases and no jump is necessary.

(3) The continuous growth of a partially non cohesive crack. Once the upper part of the loop has been reached, which can occur after a jump, the crack evolution simply follows that upper part of the loop in the direction of increasing time and finally the crack length will tends to $a_{c}$ when $t$ goes to infinity as in the perfect case. The system will finally forget its initial imperfection.

All these results can be seen on Fig. 8 where are considered five cases of imperfection size. The first three, which correspond to a small initial crack length, lead to a jump whereas the last two, corresponding to a sufficiently large initial crack length, give rise to a continuous growth of the crack. Of course, the critical length of the initial crack above which the evolution is continuous depends both on $\ell$ and $d_{c}$. In any case, one sees the fundamental role played by the loop of the perfect system.

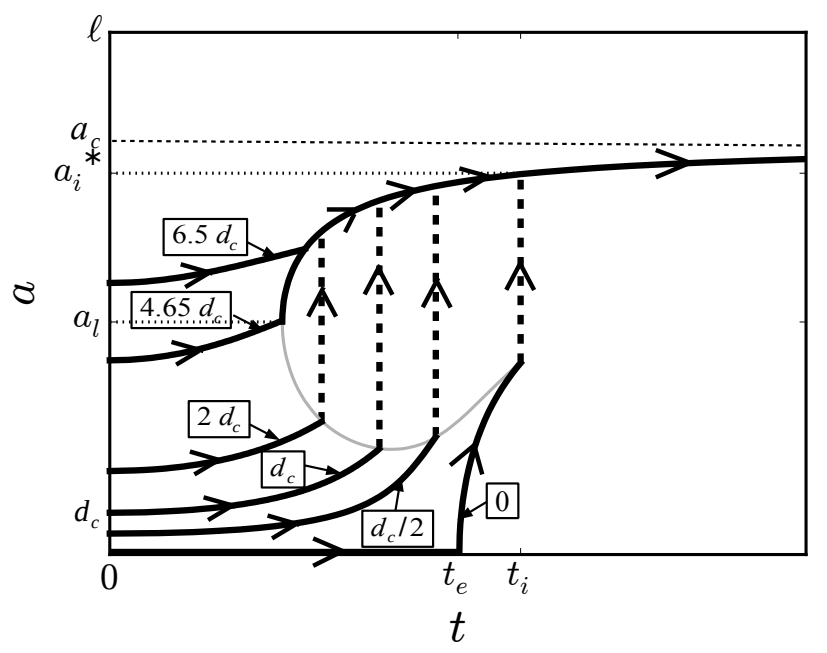

Fig. 8. Evolution of the crack length $a$ under a monotonically increasing loading for different lengths $a_{0}$ of the centered initial non cohesive crack. Here, $d_{c} / \ell=0.1$ and $a_{0}=$ $0, d_{c} / 2, d_{c}, 2 d_{c}, 4.65 d_{c}, 6 d_{c}$

\section{BARENBLATT'S COHESIVE CRACK EVOLUTION}

The aim of this section is to generalise the previous results by supposing that the crack evolution is governed by Barenblatt's cohesive model. The model assumes that the normal cohesive stress $\sigma_{n n}$ is no more a constant but a continuous function of displacement jump $\left[u_{2}\right]$. This assumption leads to integro-differential equations in the resolution of the crack evolution problem where a semi-analytical method using Chebychev polynomials developed in [15] is necessary. We recall some principal formulations of 
Barenblatt's cohesive model, then formulate the generalised crack evolution problem in two stages: the first one of purely cohesive crack and the second one of partially noncohesive crack. Some important results of the dimensionless resolution are presented for a special case of linear Barenblatt's cohesive model.

\subsection{Barenblatt's model of crack opening}

This model is based on the principal assumption that the normal stress $\sigma_{n n}$ giving the interaction between the crack lips is a continuous, monotonically decreasing function of displacement jump along the cohesive zone, i.e. $\left.\left[u_{n}\right]\right] \geq \delta_{c}$, while the Dugdale's model assumes this physical quantity is constant in this zone (see (9)). Specifically, we have

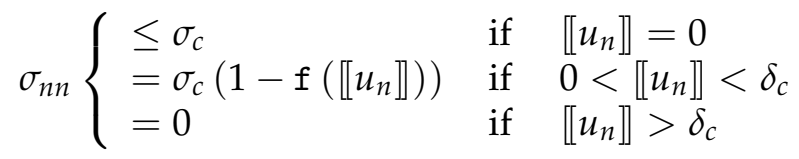

where $f$ is a monotonically increasing, positive function of $\left.\left[u_{2}\right]\right]$ which satisfies the following conditions

$$
\left.\mathrm{f}(0)=0, \quad \mathrm{f}\left(\left[\left[u_{n}\right]\right]\right)=1 \forall\left[\left[u_{n}\right]\right] \geq \delta_{c}, \quad \mathrm{f}^{\prime}\left(\left[u_{n}\right]\right]\right) \geq 0, \quad \forall \llbracket\left[u_{n}\right] \geq 0 .
$$

Consequently, the surface energy density reads as

$$
\Phi\left(\llbracket u_{n} \rrbracket\right)=\left\{\begin{array}{lll}
\infty+ & \text { if } & \llbracket u_{n} \rrbracket=0 \\
\sigma_{c}\left[\llbracket u_{n} \rrbracket-\int_{0}^{\llbracket u_{n} \rrbracket} \mathrm{f}(s) \mathrm{d} s\right] & \text { if } & 0<\llbracket\left[u_{n} \rrbracket<\delta_{c}\right. \\
G_{c} & \text { if } & \llbracket u_{n} \rrbracket>\delta_{c}
\end{array}\right.
$$

The relationship between the critical cohesive stress $\sigma_{c}$ and the critical energy release rate $G_{c}$ can be written as follows

$$
G_{c}=\sigma_{c}\left[\delta_{c}-\int_{0}^{\delta_{c}} \mathbf{f}(s) \mathrm{d} s\right] .
$$

Fig. 9 shows the Barenblatt's surface energy density and the cohesive stress in function of the jump displacement. As in the Dugdale's case, the cracks are generally divided into two zones: the cohesive zone and the non cohesive zone.
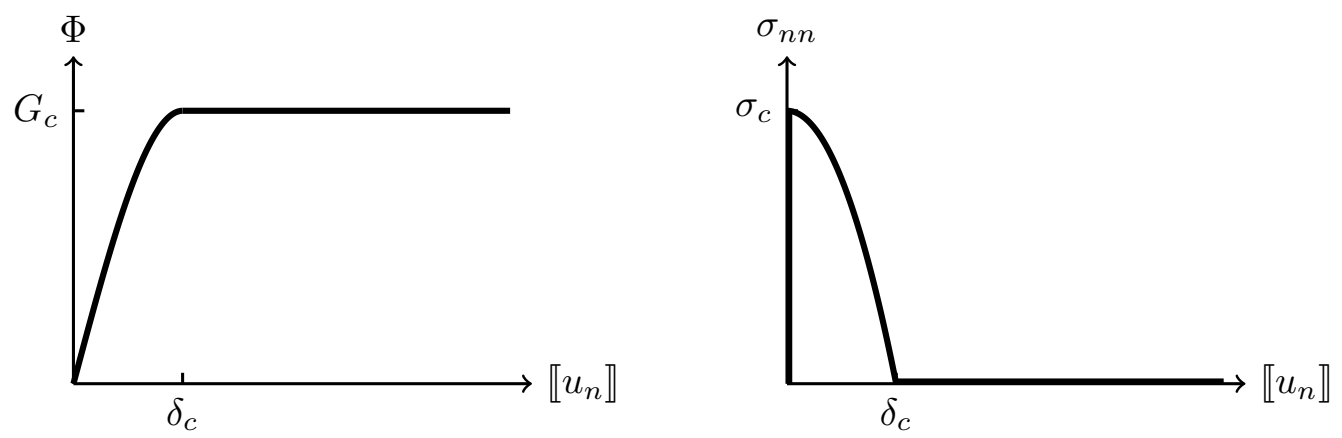

Fig. 9. Barenblatt's the surface energy density and the cohesive stress in function of jump displacement 
Recalling that the problem settings and the assumptions presented in Section 2 remain the same. In addition, the three types of crack state in Barenblatt's case are always shown in Fig. 3: no crack, full cohesive crack and partially non cohesive crack. One obtains the same elastic response as in Dugdale's case where the normal stress distribution along $\Gamma$ is given by (6) and the time corresponding to the nucleation of cohesive crack is still $t_{e}$. The two scale approach can always be used to study Barenblatt's crack evolution by assuming the hierarchy of the length, i.e. $d \ll L, \ell \lesssim L$. The equations are formulated in the Barenblatt's general case, then a dimensionless study will be detailed for a particular case of linear cohesive law.

\subsection{Formulations of two-scale approach for Barenblatt's general cohesive model}

\subsubsection{Fully cohesive crack}

Considering a fully cohesive with length $2 a>0$ at time $t>0$. For given $a$ and $t$, we define the associated displacement and stress fields as the unique solution, denoted $(\mathbf{u}[t, a], \sigma[t, a])$, of the following linear elastic problem posed on the cracked body with uniform cohesive forces on the crack lips

$$
\left\{\begin{array}{lll}
\operatorname{div} \sigma[t, a]=0 & \text { in } \Omega \backslash([-a, a] \times\{0\}) \\
\sigma[t, a]=\lambda \operatorname{tr}(\varepsilon(\mathbf{u}[t, a])) \mathbf{I}+2 \mu \varepsilon(\mathbf{u}[t, a]) & \text { in } \Omega \backslash([-a, a] \times\{0\}) \\
\mathbf{u}[t, a]=t \mathbf{U} & \text { on } \partial_{D} \Omega \\
\sigma[t, a] \mathbf{n}=t \mathbf{F} & \text { on } \partial_{N} \Omega \\
\sigma[t, a] \mathbf{e}_{2}=\sigma_{c}\left(1-\mathrm{f}\left(\left[u(t)_{2}\right]\left(x_{1}\right)\right)\right) \mathbf{e}_{2} & \text { on }[-a, a] \times\{0\}
\end{array}\right.
$$

In the same way as Dugdale's case, one introduces in (47) the gaps between the solution with the elastic fields, i.e.

$$
\overline{\mathbf{u}}[t, a]=\mathbf{u}[t, a]-t \mathbf{u}^{\mathrm{el}}, \quad \bar{\sigma}[t, a]=\sigma[t, a]-t \sigma^{\mathrm{el}},
$$

where $\bar{\sigma}[t, a](\mathbf{x})$ should tend to 0 when $\|\mathbf{x}\|$ becomes large by comparison with $a$. In addition, the gap of the normal stress verifies $\bar{\sigma}[t, a]_{22}\left(x_{1}\right)=\sigma_{c}\left(1-\mathrm{f}\left(\left[u(t)_{2}\right]\right)\right)-t \Sigma\left(x_{1}\right)$ where $\Sigma\left(x_{1}\right)$ is given by (6). Consequently, we obtain

$$
\left.\bar{\sigma}[t, a]_{22}\left(x_{1}\right)=\left(1-\frac{t}{t_{e}}\right) \sigma_{c}-\mathrm{f}\left(\llbracket u(t)_{2}\right]\left(x_{1}\right)\right) \sigma_{c}+2 \frac{t}{t_{e}} \frac{x_{1}^{2}}{\ell^{2}} \sigma_{c}+o\left(x_{1}^{2}\right), \quad\left|x_{1}\right|<a .
$$

By expanding the normal stress distribution up to the second order, one can write the problem giving the gaps $(\overline{\mathbf{u}}, \bar{\sigma})$ in a neighborhood of the origin as follows

$$
\begin{cases}\operatorname{div} \bar{\sigma}[t, a]=0 & \text { in } \mathbb{R}^{2} \backslash([-a, a] \times\{0\}) \\ \bar{\sigma}[t, a]=\lambda \operatorname{tr}(\varepsilon(\mathbf{u}[t, a])) \mathbf{I}+2 \mu \varepsilon(\overline{\mathbf{u}}[t, a]) & \text { in } \mathbb{R}^{2} \backslash([-a, a] \times\{0\}) \\ \bar{\sigma}[t, a](\mathbf{x}) \rightarrow 0 & \text { when }\|\mathbf{x}\| \rightarrow \infty \\ \left.\bar{\sigma}[t, a]\left(x_{1}, 0\right) \mathrm{e}_{2}=\left(1-\mathrm{f}\left(\llbracket u(t)_{2}\right]\left(x_{1}\right)\right)-\frac{t}{t_{e}}+2 \frac{t}{t_{e}} \frac{x_{1}^{2}}{\ell^{2}}\right) \sigma_{c} \mathbf{e}_{2} & \text { when } \quad x_{1} \in(-a, a)\end{cases}
$$

This problem can always be solved with the method of complex potentials developed by [19]. Here, the method is applied with the following normal stress distribution:

$$
\mathrm{T}\left(x_{1}\right)=\left(1-\mathrm{f}\left(\left[u(t)_{2}\right]\left(x_{1}\right)\right)-\frac{t}{t_{e}}+2 \frac{t}{t_{e}} \frac{x_{1}^{2}}{\ell^{2}}\right) \sigma_{c} .
$$


By integrating, the stress intensity factor at the crack tips reads as

$$
\mathrm{K}_{\mathrm{I}}[t, a]=\sigma_{c} \sqrt{\pi a}\left(\left(1-\frac{a^{2}}{\ell^{2}}\right) \frac{t}{t_{e}}-1\right)+\frac{\sigma_{c}}{\sqrt{\pi a}} \int_{-a}^{a} \mathrm{f}\left(\left[\left[u(t)_{2}\right](s)\right) \sqrt{\frac{a+s}{a-s}} \mathrm{~d} s .\right.
$$

The condition $\mathrm{K}_{\mathrm{I}}[t, a]=0$ gives us an implicit relationship between the time $t$, the position of the non cohesive zone tips $a$ and the fully cohesive crack opening:

$$
\pi a\left(1-\left(1-\frac{a^{2}}{\ell^{2}}\right) \frac{t}{t_{e}}\right)=\int_{-a}^{a} \mathrm{f}\left(\left[u(t)_{2}\right](s)\right) \sqrt{\frac{a+s}{a-s}} \mathrm{~d} s .
$$

This equation is valid as long as the crack opening at $x_{1}=0$ if less than $\delta_{c}$. The complex potential jump $\varphi(z)$ through the fully cohesive crack must be considered to study the crack opening. By using the normal stress distribution (49) in the calculation, the derivative of the complex potential can be expressed as follows

$$
\begin{aligned}
\varphi^{\prime}(z)= & \frac{\sigma_{c}}{2}\left(\frac{t}{t_{e}}-1\right)\left(\frac{z}{\sqrt{z^{2}-a^{2}}}-1\right)+\frac{\sigma_{c}}{2} \frac{t}{t_{e}}\left(-\frac{2 z \sqrt{z^{2}-a^{2}}}{\ell^{2}}+\frac{2 z^{2}}{\ell^{2}}-\frac{a^{2} z}{\ell^{2} \sqrt{z^{2}-a^{2}}}\right) \\
& -\sigma_{c} \frac{\chi(z)}{2 i \pi} \int_{S_{\mathbf{u}}} \frac{\mathrm{f}\left(\llbracket u(t)_{2} \rrbracket(\zeta)\right)}{\chi\left(\zeta^{+}\right)(\zeta-z)} \mathrm{d} \zeta .
\end{aligned}
$$

In this formulation, $S_{\mathbf{u}}=[-a, a] \times\{0\}$ denotes the set of discontinuity points of displacement field $\mathbf{u}$ and $\chi$ is a complex function defined on $\mathbb{C} \backslash S_{\mathbf{u}}$

$$
\chi:= \begin{cases}\mathbb{C} \backslash S_{\mathbf{u}} & \rightarrow \mathbb{C} \\ z & \mapsto \chi(z)=\frac{1}{\sqrt{z^{2}-a^{2}}}\end{cases}
$$

We deduce the complex potential jump as follows

$$
\begin{aligned}
\llbracket \varphi \rrbracket\left(x_{1}\right)= & -\sigma_{c}\left(\frac{t}{t_{e}}-1\right) \frac{i x_{1}}{\sqrt{a^{2}-x_{1}^{2}}}+\sigma_{c} \frac{t}{t_{e}} \mathrm{i} \frac{x_{1}\left(2 x_{1}^{2}-a^{2}\right)}{\ell^{2} \sqrt{a^{2}-x_{1}^{2}}} \\
& +\sigma_{c} \frac{i}{\pi \sqrt{a^{2}-x_{1}^{2}}} \int_{-a}^{a} \mathrm{f}\left(\llbracket u(t)_{2} \rrbracket(s)\right) \frac{\sqrt{a^{2}-s^{2}}}{s-x_{1}} \mathrm{~d} s .
\end{aligned}
$$

By using the relationship between the jumps of the complex potentials and of the normal displacement, this expression leads to

$$
\begin{aligned}
\llbracket\left[u(t)_{2}\right]^{\prime}\left(x_{1}\right)= & -4\left(1-v^{2}\right) \frac{\sigma_{c}}{E}\left(\frac{t}{t_{e}}-1\right) \frac{x_{1}}{\sqrt{a^{2}-x_{1}^{2}}} \\
& +4\left(1-v^{2}\right) \frac{\sigma_{c}}{E} \frac{t}{\ell_{e}} \frac{x_{1}\left(2 x_{1}^{2}-a^{2}\right)}{\ell^{2} \sqrt{a^{2}-x_{1}^{2}}} \\
& +\frac{4\left(1-v^{2}\right)}{\pi \sqrt{a^{2}-x_{1}^{2}}} \frac{\sigma_{c}}{E} \int_{-a}^{a} \mathrm{f}\left(\left[u(t)_{2}\right](s)\right) \frac{\sqrt{a^{2}-s^{2}}}{s-x_{1}} \mathrm{~d} s .
\end{aligned}
$$


The study of the fully cohesive crack evolution, i.e. the crack length $a(t)$ and the crack opening $\left.\left[u(t)_{2}\right]\right]$ in function of the time $t$, consists in solving the two integro-differential equations (50) and (53).

\subsubsection{Partially non cohesive crack}

Let us consider now the partially non cohesive crack evolution at time $t$ whose non cohesive length is $2 b$ and whose cohesive zone tips are at $\pm a$. For given $(a, b, t)$ with $0<b<a$ and $t>0$, we define the associated displacement and stress fields as the unique solution, denoted $(\mathbf{u}[t, a, b], \sigma[t, a, b])$, of a linear elastic problem posed on the cracked body with non uniform cohesive forces on the crack lips. By using the same perturbation method as the fully cohesive crack calculation, one considers the following problem giving the gap fields in a neighborhood of the origin $\mathbf{O}$

$$
\begin{cases}\operatorname{div} \bar{\sigma}[t, a, b]=\mathbf{0} & \text { in } \mathbb{R}^{2} \backslash([-a, a] \times\{0\}) \\ \bar{\sigma}[t, a, b]=\lambda \operatorname{tr}(\varepsilon[t, a, b])) \mathbf{I}+2 \mu \varepsilon(\overline{\mathbf{u}}[t, a, b]) & \text { in } \mathbb{R}^{2} \backslash([-a, a] \times\{0\}) \\ \bar{\sigma}[t, a, b](\mathbf{x}) \rightarrow 0 & \text { when }|\mathbf{x}| \rightarrow \infty \\ \bar{\sigma}[t, a, b]\left(x_{1}, 0\right) \mathbf{e}_{2}=\mathrm{T}\left(x_{1}\right) \mathbf{e}_{2} & \text { sur }(-a, a) \times\{0\}\end{cases}
$$

where the cohesive force distribution is given by

$$
\mathrm{T}\left(x_{1}\right)=\left\{\begin{array}{lll}
\left(-\frac{t}{t_{e}}+2 \frac{t}{t_{e}} \frac{x_{1}^{2}}{\ell^{2}}\right) \sigma_{c} & \text { if } & \left|x_{1}\right|<b \\
\left(1-\mathrm{f}\left(\llbracket u(t)_{2} \rrbracket\left(x_{1}\right)\right)-\frac{t}{t_{e}}+2 \frac{t}{t_{e}} \frac{x_{1}^{2}}{\ell^{2}}\right) \sigma_{c} & \text { if } & b<\left|x_{1}\right|<a
\end{array}\right.
$$

At a given time $t>0, a$ and $b$ must satisfy the two necessary conditions (see Propositions 2.2 and 2.1)

$$
\left\{\begin{array}{l}
\mathrm{K}_{\mathrm{I}}[t, a, b]=0 \\
\left.\left[u(t, a, b)_{2}\right]\right](b)=\delta_{c}
\end{array}\right.
$$

By using the normal stress distribution (55) in the calculation of the stress intensity factor, we obtain

$$
\begin{aligned}
\mathrm{K}_{1}[t, a, b]= & \sigma_{c} \sqrt{\pi a}\left(\left(1-\frac{a^{2}}{\ell^{2}}\right) \frac{t}{t_{e}}-1+\frac{2}{\pi} \arcsin \frac{b}{a}\right) \\
& \left.+\frac{\sigma_{c}}{\sqrt{\pi a}}\left(\int_{-a}^{a} \mathrm{f}\left(\llbracket u(t)_{2}\right](s)\right) \sqrt{\frac{a+s}{a-s}} \mathrm{~d} s-\int_{-b}^{b} \mathrm{f}\left(\llbracket u(t)_{2} \rrbracket(s)\right) \sqrt{\frac{a+s}{a-s}} \mathrm{~d} s .\right)
\end{aligned}
$$

The condition $\mathrm{K}_{\mathbf{l}}[t, a, b]=0$ gives the first implicit relationship between $(a, b, t)$

$$
\left.\pi a\left(\frac{2}{\pi} \arccos \frac{b}{a}-\left(1-\frac{a^{2}}{\ell^{2}}\right) \frac{t}{t_{e}}\right)=\int_{-a}^{a} \mathrm{f}\left(\left[u(t)_{2}\right]\right]\left(x_{1}\right)\right) \sqrt{\frac{a+s}{a-s}} \mathrm{~d} s-\int_{-b}^{b} \mathrm{f}\left(\left[u(t)_{2}\right]\left(x_{1}\right)\right) \sqrt{\frac{a+s}{a-s}} \mathrm{~d} .
$$

We consider now the normal displacement jump $[u(t, a, b)]$. Without detailing the integral steps, we write directly in the following equation the implicit expression of the 
complex potential jump $\llbracket \varphi]^{\prime}\left(x_{1}\right)$

$$
\begin{aligned}
\llbracket \varphi \rrbracket^{\prime}\left(x_{1}\right)= & -\frac{\mathrm{i} \sigma_{c} x_{1}}{\sqrt{a^{2}-x_{1}^{2}}}\left(\frac{t}{t_{e}}\left(1-\frac{a^{2}}{\ell^{2}}\right)-\frac{2}{\pi} \arccos \frac{b}{a}\right) \\
& -2 \mathrm{i} \sigma_{c}\left(\frac{x_{1} \sqrt{a^{2}-x_{1}^{2}}}{\ell^{2}} \frac{t}{t_{e}}+\frac{1}{\pi} \operatorname{arctanh}\left(\frac{b \sqrt{a^{2}-x_{1}^{2}}}{x_{1} \sqrt{a^{2}-b^{2}}}\right)\right) \\
& +\frac{i \sigma_{c}}{\pi \sqrt{a^{2}-x_{1}^{2}}}\left(\int_{-a}^{a} \mathrm{f}\left(\llbracket u(t)_{2} \rrbracket\left(x_{1}\right)\right) \frac{\sqrt{a^{2}-s^{2}}}{s-x_{1}} \mathrm{~d} s-\int_{-b}^{b} \mathrm{f}\left(\llbracket u(t)_{2} \rrbracket\left(x_{1}\right)\right) \frac{\sqrt{a^{2}-s^{2}}}{s-x_{1}} \mathrm{~d} s\right) .
\end{aligned}
$$

Finally, the relationship between thejumps of the normal displacement and of the complex potential gives us the following integro-differential equation for $u(t)_{2}$

$$
\begin{aligned}
\left.\llbracket u(t)_{2}\right]^{\prime}\left(x_{1}\right)= & -\frac{4\left(1-v^{2}\right) \sigma_{c} x_{1}}{E \sqrt{a^{2}-x_{1}^{2}}}\left(\frac{t}{t_{e}}\left(1-\frac{a^{2}}{\ell^{2}}\right)-\frac{2}{\pi} \arccos \frac{b}{a}\right) \\
& -8\left(1-v^{2}\right) \frac{\sigma_{c}}{E}\left(\frac{x_{1} \sqrt{a^{2}-x_{1}^{2}}}{\ell^{2}} \frac{t}{t_{e}}+\frac{1}{\pi} \operatorname{arctanh}\left(\frac{b \sqrt{a^{2}-x_{1}^{2}}}{x_{1} \sqrt{a^{2}-b^{2}}}\right)\right) \\
& \left.+\frac{4\left(1-v^{2}\right) \sigma_{c}}{\pi E \sqrt{a^{2}-x_{1}^{2}}}\left(\int_{-a}^{a} \mathrm{f}\left(\left[u(t)_{2}\right]\left(x_{1}\right)\right) \frac{\sqrt{a^{2}-s^{2}}}{s-x_{1}} \mathrm{~d} s-\int_{-b}^{b} \mathrm{f}\left(\llbracket u(t)_{2}\right]\left(x_{1}\right)\right) \frac{\sqrt{a^{2}-s^{2}}}{s-x_{1}} \mathrm{~d} s\right) .
\end{aligned}
$$

The dimensionless semi-analytical solution of the integro-differential equations are considered in the simple case of a linear Barenblatt's cohesive law.

\subsection{Particular case of a linear Barenblatt's cohesive law}

One assumes for this particular case that the cohesive normal stress $\sigma_{n n}$ is a linear decreasing function of the normal displacement jump $\left[\left[u_{n}\right]\right]$ as long as $0<\left[\left[u_{n}\right]\right]<\delta_{c}$

$$
\sigma_{n n}\left\{\begin{array}{llll}
\leq \sigma_{c} & \text { if } & & \left.\left[u_{n}\right]\right]=0 \\
=\sigma_{c}\left(1-\frac{\left.\llbracket u_{n}\right]}{\delta_{c}}\right) & \text { if } & 0<\llbracket\left[u_{n} \rrbracket<\delta_{c}\right. \\
=0 & \text { if } & {\left[u_{n}\right]>\delta_{c}}
\end{array}\right.
$$

We deduce the expression of the surface energy density in function of the normal displacement jump

$$
\Phi\left(\llbracket\left[u_{n} \rrbracket\right)=\left\{\begin{array}{lll}
\infty+ & \text { if } & \llbracket u_{n} \rrbracket<0 \\
\sigma_{c} \llbracket\left[u_{n} \rrbracket\left(1-\frac{\llbracket u_{n} \rrbracket}{2 \delta_{c}}\right)\right. & \text { if } & 0 \leq \llbracket u_{n} \rrbracket<\delta_{c} \\
G_{c} & \text { if } & \llbracket u_{n} \rrbracket>\delta_{c}
\end{array}\right.\right.
$$


The Barenblatt's crack evolution in two stages (first fully cohesive, then partially non cohesive) can be studied now by using this simplified cohesive law.

\subsubsection{Fully cohesive crack}

The relationship (50) becomes

$$
\pi a\left(1-\left(1-\frac{a^{2}}{\ell^{2}}\right) \frac{t}{t_{e}}\right)=\int_{-a}^{a} \frac{\llbracket u(t)_{2} \rrbracket(s)}{\delta_{c}} \sqrt{\frac{a+s}{a-s}} \mathrm{~d} .
$$

The expression of the derivative of the displacement jump (53) can be rewritten as follows

$$
\begin{aligned}
{\left[\left[u(t)_{2}\right]^{\prime}\left(x_{1}\right)=\right.} & -4\left(1-v^{2}\right) \frac{\sigma_{c}}{E} \frac{t}{t_{e}}\left(1-\frac{t_{e}}{t}\right) \frac{x_{1}}{\sqrt{a^{2}-x_{1}^{2}}} \\
& +4\left(1-v^{2}\right) \frac{\sigma_{c}}{E} \frac{t}{t_{e}} \frac{x_{1}\left(2 x_{1}^{2}-a^{2}\right)}{\ell^{2} \sqrt{a^{2}-x_{1}^{2}}} \\
& +\frac{4\left(1-v^{2}\right)}{\pi \sqrt{a^{2}-x_{1}^{2}}} \frac{\sigma_{c}}{E} \int_{-a}^{a} \frac{\left[u(t)_{2}\right](s)}{\delta_{c}} \frac{\sqrt{a^{2}-s^{2}}}{s-x_{1}} \mathrm{~d} s .
\end{aligned}
$$

The crack tip position $a(t)$ and the crack opening $\left.\left[u(t)_{2}\right]\right]$ are solutions of the integrodifferential system of equations (60) and (61) in the case of the linear Barenblatt's cohesive law. Let us introduce the following dimensionless variables

$$
\tilde{x}_{1}:=\frac{x_{1}}{a} \in[-1,1], \quad \bar{\delta}_{t}:=\frac{\left.\left[u(t)_{2}\right]\right]}{\frac{4 \lambda}{\pi^{2}} \frac{a^{2}}{\ell^{2}} \frac{t}{t_{e}} \delta_{c}}
$$

where $\lambda:=\frac{\pi^{2}}{4} \frac{a}{d_{c}}$ is a dimensionless variable proportional to crack length. By using these variables, Eq. (60) can be simplified as follows

$$
\pi\left(1-\frac{\ell^{2}}{a^{2}}\left(1-\frac{t_{e}}{t}\right)\right)=\frac{4 \lambda}{\pi^{2}} \int_{-1}^{1} \bar{\delta}_{t}(\tilde{s}) \sqrt{\frac{1+\tilde{s}}{1-\tilde{s}}} \mathrm{~d} \tilde{s}
$$

We obtain also a dimensionless form of the integro-differential equation (61)

$$
\begin{aligned}
\frac{2}{\pi} \bar{\delta}_{t}^{\prime}\left(\tilde{x}_{1}\right)= & \frac{\tilde{x}_{1}}{\sqrt{1-\tilde{x}_{1}^{2}}}\left[-\frac{\ell^{2}}{a^{2}}\left(1-\frac{t_{e}}{t}\right)+\left(2 \tilde{x}_{1}^{2}-1\right)\right] \\
& +\frac{1}{\pi \sqrt{1-\tilde{x}_{1}^{2}}} \frac{4 \lambda}{\pi^{2}} \int_{-1}^{1} \bar{\delta}_{t}(\tilde{s}) \frac{\sqrt{1-\tilde{s}^{2}}}{\tilde{s}-\tilde{x}_{1}} \mathrm{~d} \tilde{s} .
\end{aligned}
$$

This integro-differential equation can be solved semi-analytically using Chebychev polynomials developed in [15]. The expression of the dimensionless displacement jump $\bar{\delta}_{t}$ of the Barenblatt's fully cohesive crack depends on the crack length $a$ which increases with the loading $t$. Without detailing the calculation, the dimensionless displacement 
jump $\bar{\delta}_{t}$ of the Barenblatt's fully cohesive crack corresponding to $\lambda=0,0.2,1,2$ in comparison with Dugdale's case is shown in Fig. 10.

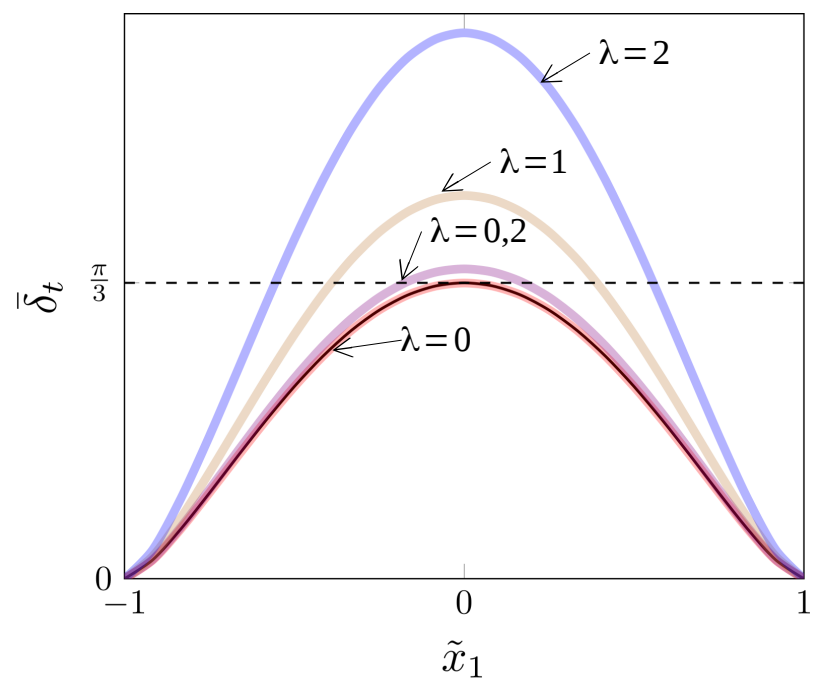

Fig. 10. The dimensionless displacement jump $\bar{\delta}_{t}$ of the Barenblatt's fully cohesive crack corresponding to $\lambda=0,0.2,1,2$ in comparison with Dugdale's case (black curve)

Let us now rewrite the results of Dugdale's crack evolution in dimensionless form and compare with the Barenblatt's crack corresponding to different crack lengths.

- Dimensionless calculations of Dugdale's fully crack evolution. The dimensionless variables defined in (62) allow us to express the dimensionless normal displacement of Dugdale's fully cohesive crack as follows

$$
\bar{\delta}_{t}\left(\tilde{x}_{1}\right)=\frac{\pi}{3}\left(1-\tilde{x}_{1}^{2}\right)^{3 / 2} .
$$

Consequently, the formulation of the dimensionless normal displacement remains always the same during the Dugdale's fully cohesive crack evolution. In particular, $\bar{\delta}_{t}\left(\tilde{x}_{1}\right)$ is decreasing with $\tilde{x}_{1}$, equals to 0 for $\tilde{x}_{1}= \pm 1$ and reaches its maximal values $\pi / 3$ for $\tilde{x}_{1}=0$. Besides, the non cohesive crack appears when $\left[\left[u(t)_{2}\right]\right](0)$ reaches the critical value $\delta_{c}$. The loading $t_{i}$ corresponding to the end of fully cohesive phase can be calculated by the following equation

$$
\frac{\pi}{3} a^{3}\left(t_{i}\right) t_{i}=d_{c} \ell^{2} t_{e}
$$

- Barenblatt's fully cohesive crack of very small length. During this phase, the crack length $a$ is very close to the material characteristic length $d_{c}$, i.e. the dimensionless variable $\lambda$ is very close to 0 . Because of a very small fully cohesive crack opening, the cohesive stress along the crack is quasi-uniform and very close to the critical value $\sigma_{c}$. In consequence, the results obtained in this phase of Barenblatt's fully cohesive crack can be well approximated by Dugdale's results. This good approximation is confirmed by Fig. 10. 
- Barenblatt's fully cohesive crack of large length. When the crack length $a$ is at the same order or much greater than $d_{c}$, i.e. when $\lambda \gtrsim 1$, the curves of the dimensionless displacement jump $\bar{\delta}_{t}$ corresponding to different values of $\lambda$ are shown in Fig. 10. In particular, $\bar{\delta}_{t}\left(\tilde{x}_{1}\right)$ is a decreasing function of $\left|\tilde{x}_{1}\right|$, is negligible for $\tilde{x}_{1}= \pm 1$ and reaches its maximal value (which is always greater than the maximum $\pi / 3$ of Dugdale's crack) for $\tilde{x}_{1}=0$. Besides, the loading $t_{i}$ corresponding to the end of the fully cohesive phase is now given by

$$
\bar{\delta}_{t_{i}^{*}}(0) a^{3}\left(t_{i}^{*}\right) t_{i}^{*}=d_{c} \ell^{2} t_{e} .
$$

\subsubsection{Partially non cohesive crack}

By using the linear Barenblatt's cohesive law assumptions, the implicit relationship (57) between $(a b, b, t)$ becomes

$$
\pi a\left(\frac{2}{\pi} \arccos \frac{b}{a}-\left(1-\frac{a^{2}}{\ell^{2}}\right) \frac{t}{t_{e}}\right)=\int_{-a}^{a} \frac{\llbracket u(t)_{2} \rrbracket(s)}{\delta_{c}} \sqrt{\frac{a+s}{a-s}} \mathrm{~d} s-\int_{-b}^{b} \frac{\llbracket u(t)_{2} \rrbracket(s)}{\delta_{c}} \sqrt{\frac{a+s}{a-s}} \mathrm{~d} s .
$$

The integro-differential equation giving the normal displacement jump can be rewritten as follows

$$
\begin{aligned}
\left.\llbracket u(t)_{2}\right]^{\prime}\left(x_{1}\right)= & -\frac{4\left(1-v^{2}\right) \sigma_{c} x_{1}}{E \sqrt{a^{2}-x_{1}^{2}}}\left(\frac{t}{t_{e}}\left(1-\frac{a^{2}}{\ell^{2}}\right)-\frac{2}{\pi} \arccos \frac{b}{a}\right) \\
& -8\left(1-v^{2}\right) \frac{\sigma_{c}}{E}\left(\frac{x_{1} \sqrt{a^{2}-x_{1}^{2}}}{\ell^{2}} \frac{t}{t_{e}}+\frac{1}{\pi} \operatorname{arctanh}\left(\frac{b \sqrt{a^{2}-x_{1}^{2}}}{x_{1} \sqrt{a^{2}-b^{2}}}\right)\right) \\
& +\frac{4\left(1-v^{2}\right) \sigma_{c}}{\pi E \sqrt{a^{2}-x_{1}^{2}}}\left(\int_{-a}^{a} \frac{\left[u(t)_{2}\right](s)}{\delta_{c}} \frac{\sqrt{a^{2}-s^{2}}}{s-x_{1}} \mathrm{~d} s-\int_{-b}^{b} \frac{\left[u(t)_{2}\right](s)}{\delta_{c}} \frac{\sqrt{a^{2}-s^{2}}}{s-x_{1}} \mathrm{~d} s\right) .
\end{aligned}
$$

By injecting the dimensionless variables defined in (62) and setting $\alpha=\frac{b}{a} \in(0,1)$ in (66), we obtain

$$
\frac{\pi^{3}}{4 \lambda}\left(\frac{2}{\pi} \frac{\ell^{2}}{a^{2}} \frac{t_{e}}{t} \arccos \alpha+1-\frac{\ell^{2}}{a^{2}}\right)=\int_{-1}^{1} \bar{\delta}_{t}(\tilde{s}) \sqrt{\frac{1+\tilde{s}}{1-\tilde{s}}} \mathrm{~d} \tilde{s}-\int_{-\alpha}^{\alpha} \bar{\delta}_{t}(\tilde{s}) \sqrt{\frac{1+\tilde{s}}{1-\tilde{s}}} \mathrm{~d} \tilde{s}
$$

The integro-differential equation (67) can be also rewritten under a dimensionless form

$$
\begin{aligned}
\frac{2}{\pi} \bar{\delta}_{t}^{\prime}\left(\tilde{x}_{1}\right)= & \frac{2}{\pi} \frac{\ell^{2}}{a^{2}} \frac{t_{e}}{t} \arccos \alpha+1-\frac{\ell^{2}}{a^{2}} \\
& -2\left(\tilde{x}_{1} \sqrt{a^{2}-\tilde{x}_{1}^{2}}+\frac{1}{\pi} \frac{\ell^{2}}{a^{2}} \frac{t_{e}}{t} \operatorname{arctanh}\left(\frac{\alpha \sqrt{1-\tilde{x}_{1}^{2}}}{\tilde{x}_{1} \sqrt{1-\alpha^{2}}}\right)\right) \\
& +\frac{4 \lambda}{\pi^{3} \sqrt{1-\tilde{x}_{1}^{2}}}\left(\int_{-1}^{1} \bar{\delta}_{t}(\tilde{s}) \frac{\sqrt{1-\tilde{s}^{2}}}{\tilde{s}-\tilde{x}_{1}} \mathrm{~d} \tilde{s}-\int_{-\alpha}^{\alpha} \bar{\delta}_{t}(\tilde{s}) \frac{\sqrt{1-\tilde{s}^{2}}}{\tilde{s}-\tilde{x}_{1}} \mathrm{~d} \tilde{s}\right) .
\end{aligned}
$$




\section{CONCLUSION}

The present paper considers the problem of the nucleation and the propagation of a cohesive crack at the tip of a notch in two-dimensional elastic structures using Dugdale's or Barenblatt's cohesive force models where the stress field associated with a pure elastic response is assumed to be smooth and bounded, but nonuniform. Further it is supposed that the material characteristic length associated with the cohesive model is small by comparison to the dimension of the body. The crack evolution can be considered in two stages: the first one where all the crack is submitted to cohesive forces, followed by a second one where a non cohesive part appears. The following results can be summarized:

- The entire crack evolution with the loading is obtained in a closed form for the Dugdale's case and in semi-analytical form for the Barenblatt's case using the method of complex potentials and a two-scale technique.

- It has been shown that the propagation is stable during the first stage, but becomes unstable with a brutal jump of the crack length as soon as the non cohesive crack part appears.

- The influence of all the parameters of the problem and sensitivity to imperfections are discussed.

\section{REFERENCES}

[1] A. A. Griffith. The phenomena of rupture and flow in solids. Philosophical Transactions of the Royal Society of London. Series A, 221, (1920), pp. 163-198. https://doi.org/10.1098/rsta.1921.0006.

[2] D. S. Dugdale. Yielding of steel sheets containing slits. Journal of the Mechanics and Physics of Solids, 8, (2), (1960), pp. 100-104. https://doi.org/10.1016/0022-5096(60)90013-2.

[3] G. I. Barenblatt. The mathematical theory of equilibrium cracks in brittle fracture. Advances in Applied Mechanics, 7, (1962), pp. 55-129. https://doi.org/10.1016/s0065-2156(08)70121-2.

[4] G. Del Piero and M. Raous. A unified model for adhesive interfaces with damage, viscosity, and friction. European Journal of Mechanics-A/Solids, 29, (4), (2010), pp. 496-507. https://doi.org/10.1016/j.euromechsol.2010.02.004.

[5] K. Keller, S. Weihe, T. Siegmund, and B. Kröplin. Generalized cohesive zone model: incorporating triaxiality dependent failure mechanisms. Computational Materials Science, 16, (1-4), (1999), pp. 267-274. https://doi.org/10.1016/s0927-0256(99)00069-5.

[6] A. Needleman. Micromechanical modelling of interfacial decohesion. Ultramicroscopy, 40, (3), (1992), pp. 203-214. https://doi.org/10.1016/0304-3991(92)90117-3.

[7] K. L. Roe and T. Siegmund. An irreversible cohesive zone model for interface fatigue crack growth simulation. Engineering Fracture Mechanics, 70, (2), (2003), pp. 209-232. https://doi.org/10.1016/s0013-7944(02)00034-6.

[8] C. Talon and A. Curnier. A model of adhesion coupled to contact and friction. European Journal of Mechanics-A/Solids, 22, (4), (2003), pp. 545-565. https://doi.org/10.1016/s09977538(03)00046-9.

[9] V. Tvergaard. Effect of fibre debonding in a whisker-reinforced metal. Materials Science and Engineering: A, 125, (2), (1990), pp. 203-213. https://doi.org/10.1016/0921-5093(90)90170-8.

[10] G. Del Piero. One-dimensional ductile-brittle transition, yielding, and structured deformations. In IUTAM Symposium on Variations of Domain and Free-Boundary Problems in Solid Mechanics. Springer, (1999), pp. 203-210. https://doi.org/10.1007/978-94-011-4738-5_24. 
[11] M. Charlotte, G. Francfort, J.-J. Marigo, and L. Truskinovsky. Revisiting brittle fracture as an energy minimization problem: comparison of Griffith and Barenblatt surface energy models. Continuous Damage and Fracture, (2000), pp. 7-18.

[12] J. Laverne and J.-J. Marigo. Approche globale, minima relatifs et Critère d'Amorçage en Mécanique de la Rupture. Comptes Rendus Mécanique, 332, (4), (2004), pp. 313-318. https://doi.org/10.1016/j.crme.2004.01.014.

[13] M. Charlotte, J. Laverne, and J.-J. Marigo. Initiation of cracks with cohesive force models: a variational approach. European Journal of Mechanics-A/Solids, 25, (4), (2006), pp. 649-669. https://doi.org/10.1016/j.euromechsol.2006.05.002.

[14] B. Bourdin, G. A. Francfort, and J.-J. Marigo. The variational approach to fracture. Journal of Elasticity, 91, (1-3), (2008), pp. 5-148. https://doi.org/10.1007/978-1-4020-6395-4.

[15] H. Ferdjani, R. Abdelmoula, J.-J. Marigo, and S. El Borgi. Study of size effects in the Dugdale model through the case of a crack in a semi-infinite plane under antiplane shear loading. Continuum Mechanics and Thermodynamics, 21, (1), (2009), pp. 41-55. https://doi.org/10.1007/s00161-009-0098-0.

[16] H. Ferdjani, R. Abdelmoula, and J.-J. Marigo. Insensitivity to small defects of the rupture of materials governed by the Dugdale model. Continuum Mechanics and Thermodynamics, 19, (3-4), (2007), pp. 191-210. https://doi.org/10.1007/s00161-007-0051-z.

[17] D. T. B. Tuyet, L. Halpern, and J.-J. Marigo. Asymptotic analysis of small defects near a singular point in antiplane elasticity, with an application to the nucleation of a crack at a notch. Mathematics and Mechanics of Complex Systems, 2, (2), (2014), pp. 141-179. https://doi.org/10.2140/memocs.2014.2.141.

[18] D. T. B. Tuyet, J. J. Marigo, and L. Halpern. Matching asymptotic method in propagation of cracks with Dugdale model. In Key Engineering Materials, Vol. 525. Trans Tech Publ, (2013), pp. 489-492.

[19] N. I. Muskhelishvili. Some basic problems of mathematical theory of elasticity. P. Noordhoff Ltd, Groningen, (1963). 